\title{
Flavonoids as an effective sensitizer for anti-cancer therapy: insights into multi-faceted mechanisms and applicability towards individualized patient profiles
}

\author{
Alena Liskova ${ }^{1} \cdot$ Marek Samec $^{1} \cdot$ Lenka Koklesova $^{1} \cdot$ Aranka Brockmueller $^{2} \cdot$ Kevin Zhai $^{3} \cdot$ Basma Abdellatif $^{3}$. \\ Manaal Siddiqui ${ }^{3} \cdot$ Kamil Biringer $^{1} \cdot$ Erik Kudela $^{1} \cdot$ Martin Pec $^{4} \cdot$ Laura Kate Gadanec $^{5} \cdot$ Miroslava Šudomová $^{6}$. \\ Sherif T. S. Hassan ${ }^{7} \cdot$ Anthony Zulli $^{5} \cdot$ Mehdi Shakibaei $^{2} \cdot$ Frank A. Giordano $^{8} \cdot$ Dietrich Büsselberg $^{3}$. \\ Olga Golubnitschaja ${ }^{9} \cdot$ Peter Kubatka ${ }^{4}$
}

Received: 13 April 2021 / Accepted: 16 April 2021 / Published online: 17 May 2021

(c) The Author(s) 2021

\begin{abstract}
Cost-efficacy of currently applied treatments is an issue in overall cancer management challenging healthcare and causing tremendous economic burden to societies around the world. Consequently, complex treatment models presenting concepts of predictive diagnostics followed by targeted prevention and treatments tailored to the personal patient profiles earn global appreciation as benefiting the patient, healthcare economy, and the society at large. In this context, application of flavonoids as a spectrum of compounds and their nano-technologically created derivatives is extensively under consideration, due to their multi-faceted anti-cancer effects applicable to the overall cost-effective cancer management, primary, secondary, and even tertiary prevention. This article analyzes most recently updated data focused on the potent capacity of flavonoids to promote anti-cancer therapeutic effects and interprets all the collected research achievements in the frame-work of predictive, preventive, and personalized (3P) medicine. Main pillars considered are:

- Predictable anti-neoplastic, immune-modulating, drug-sensitizing effects;

- Targeted molecular pathways to improve therapeutic outcomes by increasing sensitivity of cancer cells and reversing their resistance towards currently applied therapeutic modalities.
\end{abstract}

Keywords Predictive preventive personalized medicine (3PM/PPPM) - Phytochemicals $\cdot$ Flavonoids $\cdot$ Flavanones · Flavonols · Flavones · Flavanols · Isoflavonoids $\cdot$ Chalcones $\cdot$ Anthocyanidins $\cdot$ Anti-cancer agents $\cdot$ Drug-sensitizing effect $\cdot$ Targeted therapy $\cdot$ Radiotherapy $\cdot$ Chemotherapy $\cdot$ Immunotherapy $\cdot$ Therapy resistance $\cdot$ Anti-inflammation $\cdot$ Antibacterial $\cdot$ Anti-viral $\cdot$ COVID-19 $\cdot$ Signalling pathways $\cdot$ Therapy efficacy $\cdot$ Nano-carrier delivery $\cdot$ Disease management $\cdot$ Health economy $\cdot$ Health policy

Dietrich Büsselberg

dib2015@qatar-med.cornell.edu

Olga Golubnitschaja

Olga.Golubnitschaja@ukbonn.de

Peter Kubatka

peter.kubatka@uniba.sk

Extended author information available on the last page of the article

\section{Introduction}

\section{Flavonoids in primary, secondary, and tertiary anti-cancer management}

By evidence, a large portion of malignancies is considered as being preventable, and a cost-effective targeted anti-cancer protection is an issue in the overall management of the disease [1]. To this end, an application of natural substances is an attractive strategy for primary and secondary cancer prevention [2]. Preclinical cancer research demonstrates potent genoprotective properties of flavonoids in non-cancer models. Corresponding mechanisms applicable to the primary and secondary anti-cancer care include multi-faceted 
effects such as anti-Warburg cell transformation and antimitochondriopathic activities, anti-inflammatory, antibacterial, and anti-viral, among other health-beneficiary effects [3-5].

In tertiary care, flavonoids have been proposed to protect patients against poor outcomes in case of COVID-19 infection, due to their evident anti-inflammatory, anti-bacterial, and anti-viral properties $[6,7]$.

Protective anti-cancer application of flavonoids in the context of 3P medicine should follow principles of the evidence-based therapeutic effects, individualized prediction, targeted prevention, and personalization of the treatment algorithms. To this end, application of specialized analytical approaches towards a companion diagnostics is strongly recommended such as liquid biopsy analysis, risk assessment tools, multi-omics and multi-parametric analysis, and application of artificial intelligence in medicine [8].

\section{Radiotherapy and chemotherapy as hallmarks of anti-cancer treatments: status quo}

Radiotherapy and chemotherapy are hallmarks of the currently applied anti-cancer treatments [9]. Conventional anticancer strategies pose several deficits [10,11]. Despite recent progress in anti-cancer strategies, the development of resistance remains the leading cause of cancer-related mortality, and many patients develop resistance towards anti-cancer agents applied [12]. Moreover, radiotherapy, chemotherapy, and targeted therapy require effective blood flow into the tumor microenvironment; perfusion deficits reduce overall therapeutic efficacy [13]. An improved understanding of carcinogenic processes allows for the technological innovation creating more efficient therapeutic modalities [10]. Targeted anti-cancer therapies are expected to leverage unique molecular changes associated with specific cancer types $[14,15]$.

Cancer resistance can be classified into primary and acquired. Primary (or intrinsic) cancer resistance exists before the commencement of treatment [16]. It can be caused by (a) pre-existing genetic mutations in tumors resulting in decreased responsiveness to therapy, such as in the case of triple-negative breast cancer (TNBC); (b) heterogeneity of tumors with pre-existing insensitive subpopulations such as cancer stem cells (CSCs); or (c) activation of intrinsic pathways as defenses against chemotherapeutic drugs [17]. In contrast, acquired resistance develops after the initial therapy [16] and is characterized by a gradual reduction in anti-cancer efficacy. Acquired drug resistance can result from the activation of newly emerged driver genes, mutations/altered expression of drug targets, or changes in the tumor microenvironment after treatment [17]. Current statistics of the World Health Organization (WHO) indicate that one in every six deaths worldwide is due to cancer. Therefore, in a view of the consequent severe socio-economic burden to the society, it is necessary to shift the paradigm of cancer management from reactive to predictive, preventive, and personalized medical approaches $[18,19]$. Contextually, the focus of current cancer research on the identification of molecules could improve individual outcomes of cancer treatments, including overcoming resistance and increasing the sensitivity of cancer cells towards treatments applied.

Phytochemicals, isolated or in corresponding intact plants, are a verified source of natural anti-cancer molecules targeting a variety of pathways associated with neoplastic transformation and cancer progression [2, 20-41]. Indeed, flavonoids exert significant anti-cancer effects in clinical and preclinical studies [3, 42-47]. Therefore, we emphasize the great evidence-based potential of bioactive flavonoids in modulating cancer cells' response to anti-cancer drugs by overcoming resistance and/or sensitizing cancer cells to currently applied therapies.

\section{Focus of the current study: Flavonoids as a helper in anti-cancer therapy}

This review focuses on flavonoids' capacity to modulate the responsiveness of cancer to conventional treatment modalities. Of particular interest are the helper effects against cancer therapy resistance by sensitizing malignant cells towards therapies. Those properties of flavonoids clearly demonstrated in preclinical studies are considered of particularly great clinical utility, when applied to anti-cancer therapies tailored to the personalized patient profile [48-59].

\section{Source of the analyzed research data}

Data were collected from the biomedical literature sources utilizing "resistance" and "flavonoids" or "flavanones" or "flavonols " or "flavones" or "flavanols" or "isoflavonoids" or "chalcones" or "anthocyanidins" and "radiotherapy" or "chemotherapy" or "targeted therapy" or other relevant items as either keywords or medical subject heading (MeSH) terms in searches of the PubMed database. Most recently updated research published within the time-frame of 2018-2021 years has been taken into consideration for the below presented data analysis and interpretation.

\section{Anti-cancer therapy: from conventional to advanced approaches}

The development of radiotherapy to treat cancer in the early 1900s was followed by the discovery of the chemotherapy. The breakthrough of modern oncology was introduced by targeted therapies directed at specific tumor and molecular alterations and immune checkpoint inhibitors to stimulate the immune system against cancer [9]. Therefore, oncology research in the last 20 years has yielded new anti-tumor 
strategies-including monoclonal antibodies and immunotherapeutic agents- that significantly increase treatment efficacy and allow personalized, highly effective, and less toxic approaches for individual patients [9]. Unfortunately, the initial favourable response to treatment is often followed by the development of resistance that reduces therapeutic efficacy and leads to cancer relapse and recurrence [60]. However, further progress in precision medicine in the twenty-first century enables an individualized approach in the context of predictive, preventive, and personalized medicine that can improve cancer management [18, 61-66]. Cancer treatment can also be improved by applying natural substances, which sensitize cancer cells to therapeutic agents [67-70].

\section{Flavonoids: origin and classification}

lavonoids are phenolic compounds widely found in vegetables, fruits, beverages, nuts, olive oil, red wine, and medicinal plants [3, 7, 46, 47, 71, 72]. Chemically, flavonoids have fifteen-carbon skeletons consisting of two benzene rings connected through a pyrane ring. The classification of flavonoids is based on the chemical structure, oxidation level, and substitution pattern of the heterocyclic pyrane ring ( $\mathrm{C}$ ring) [7]. The classification of flavonoids is provided in Fig. 1 [3, 7, 71, 73-79].
Flavonoids exert numerous biological effects, including antioxidant, anti-inflammatory, anti-mutagenic, and anticancer activities, among others [46]. Although flavonoids have demonstrated significant anti-cancer efficacy in preclinical research [46, 69, 71, 75, 76], clinical studies evaluating the anti-cancer effects of flavonoids remain sparse. Genistein was demonstrated to be safe and tolerable in combination with chemotherapy in a recent phase I/II pilot study [43]. Other clinical trials revealed the potential of flavonoids as anticarcinogenic agents [80] and as complementary antitumor agents in colorectal cancer patients [45]. However, the significant capacity of flavonoids to improve therapeutic outcomes by improving the treatment sensitivity or reversing the resistance of cancer cells to anti-cancer therapeutic agents is currently evaluated predominantly in preclinical in vitro and in vivo research [67-70].

\section{Radiotherapy resistance}

Radiotherapy is a component of multidisciplinary treatment regimens applicable to various cancer types [81]. Up-todate technologies enable the precise delivery of radiation to tumor lesions with minimal injury to healthy tissue. However, many cancer types are associated with insensitivity to radiotherapy due to intrinsic resistance or recurrence after treatment due to acquired resistance [11]. Figure 2 provides

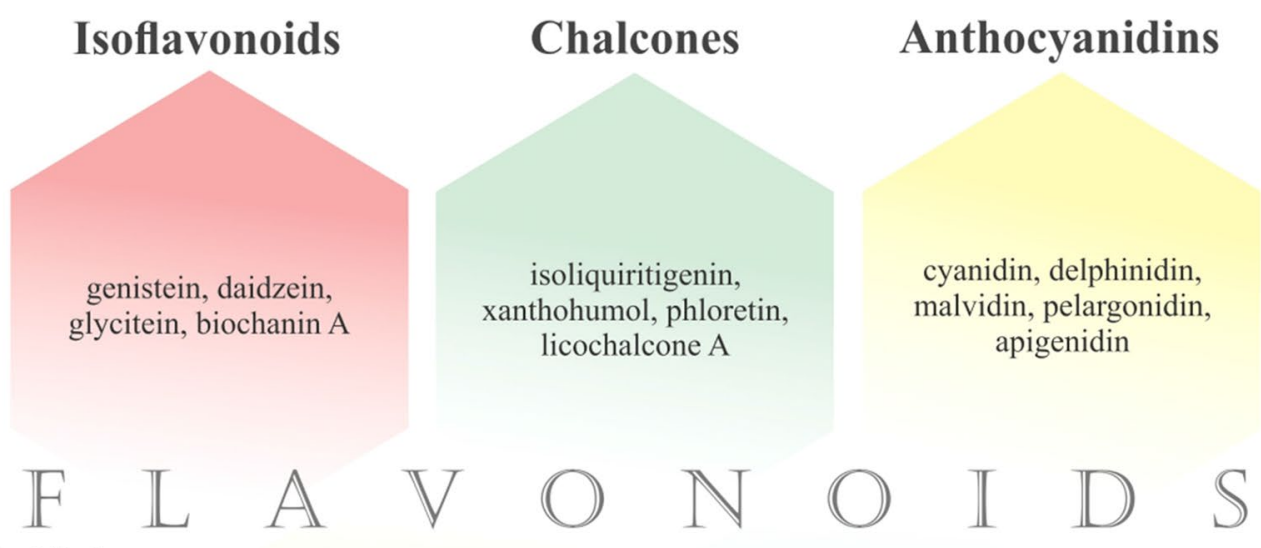

5-demethylnobiletin, apigenin, luteolin, wogonin, hispidulin, amentoflavone, scutellarin, pectolinarigenin, oroxylin, vicenin II, chrysin, cirsiliol, kaempferide, hydrate, tangeretin, morusin, 5,7-dimethoxyflavone, baicalein, diosmin, genkwanin

Flavones kaempferol, quercetin, myricetin, fisetin, rutin, tamarixetin, morin, rhamnetin, icariin

Flavonols hesperetin, hesperitin, hesperidin, taxifolin, 2'-hydroxyflavanone, liquiritigenin, eriodictyol, naringenin, naringin, bavachinin

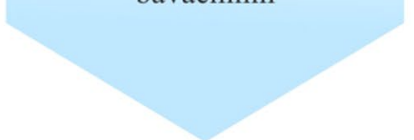

Flavanones catechin, epicatechin, epigallocatechin, epigallocatechin-3-galate

Flavanols

Fig. 1 Classification of flavonoids 


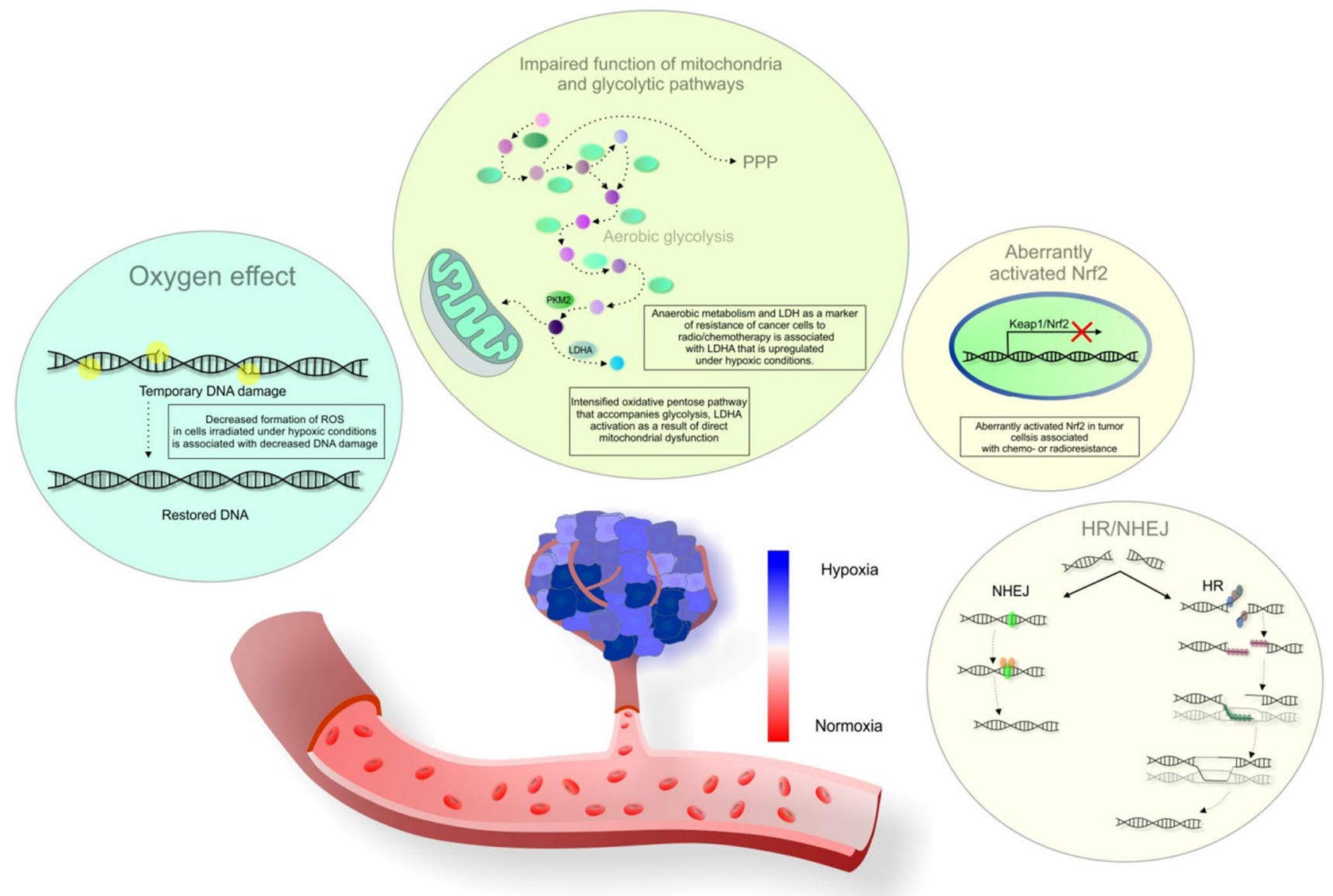

Fig. 2 Mechanisms of radiotherapy resistance of cancer cells. Explanatory notes: A hypoxic intratumoral microenvironment is a leading cause of radiotherapy failure (decreased ROS formation in irradiated cells under hypoxic conditions is associated with decreased DNA damage and the so-called "oxygen effect"). Indeed, impaired function of mitochondria and glycolytic pathways can be involved in cancer cell radioresistance (anaerobic metabolism and LDH, a marker of resistance, associated with upregulated LDHA under hypoxic conditions). LDH is a marker of perfusion-related hypoxia. Lower oxygen leads to reductions in radiation-induced ROS generation and DNA damage. Upregulation of the oxidative pentose pathway that accompanies glycolysis, activation of LDHA as a result of direct mitochondrial dysfunction or oncogene/HIF-mediated inactivation of mitochondrial function, and inhibition of pyruvate entry into mitochondria by pyruvate-dehydrogenase kinases (regulated by LDHA

a detailed overview of specific mechanisms related to cancer cells' radiotherapy resistance, including a predominantly hypoxic tumor microenvironment and the consequent modulation of mitochondrial and glycolytic pathways, Keap1/ Nrf2-related mechanisms, homologous recombination (HRR), and non-homologous end joining (NHEJ).

\section{Flavonoids sensitize cancer cells to radiotherapy}

While flavonoids have radioprotective effects on healthy cells, they are considered potent radiosensitizing molecules through HIF) are processes associated with cancer cells radioresistance [13]. Also, aberrantly activated Nrf2 in tumor cells (as a result of Keap1 or Nrf2 somatic mutations or other Keap1/Nrf2-related mechanisms) contributes to high-level resistance of cancer cells [82]. The Keapl promoter is often hypermethylated in NSCLS and leads to decreased Keap1 mRNA and protein expression; this impairs the Nrf2-Keap1 pathway (resulting in radio- and/or chemo-resistance) [83]. The homologous recombination (HRR) and non-homologous end joining (NHEJ) pathways enhance DNA repair activity and modulate cell sensitivity and resistance to radiotherapy [48]. The repair of DNA damage in dormant cancer stem cells (CSCs) is predominantly performed through NHEJ; consequently, NHEJ inhibition could overcome CSC radioresistance [84]. Indeed, CSCs are considered the primary source of resistance to radiotherapy and chemotherapy while tumor heterogeneity contributes to radiation resistance [11]. of cancer cells [85]. Genistein mediated selective radiosensitizing effects in non-small cell lung cancer (NSCLC) A549 cells by inhibiting the methylation of the Keapl gene promoter region; hypermethylation of the Keapl promoter results in chemo/radioresistance mediated by the Nrf2Keap1 pathway [83]. Also, genistein enhanced the radiosensitivity of NSCLC A549 cells, as demonstrated through increased apoptosis and Beclin-1-induced autophagy by inhibiting Bcl-xL and Bcl-xL-Beclin-1 interactions [86]. Moreover, apigenin and the terpenoid cryptotanshinone exerted synergistic radiosensitizing effects in the in vivo 
murine model of Ehrlich carcinoma, as demonstrated by the downregulation of angiogenic and lymphangiogenic regulators and the induction of apoptosis [87]. Furthermore, genistein and the tyrosine kinase inhibitor AG1024 (tyrphostin) synergistically increased the radiosensitivity of prostate cancer PC 3 and DU145 cells by suppressing the homologous recombination (HRR) and non-homologous end joining (NHEJ) pathways [48]. Breast safeguard (BSG) is a commercial nutrient supplement composed of several phytochemicals, including but not limited to flavonoids (genistein, quercetin, indol-3-carbinol, resveratrol, C-phycocyanin, gallic acid, and curcumin). BSG attenuated the responsiveness of hepatocellular carcinoma (HCC) HepG2 cells to ionizing radiation leading to the inhibition of proliferation, survival, and migration [88]. Also, quercetin pre-treatment enhanced colon cancer HT-29 and DLD-1 cells' radiosensitivity through Notch-1 and CSC targeting [89]. Moreover, Koh et al. recently evaluated the effects of baicalein on TNBC MDA-MB-231/IR cells obtained by irradiating the parental MDA-MB-231 cells with 2 Gy irradiation; MDA-MB-231 cells are characterized by enhanced migration, invasion, and stem-cell-like properties. Indeed, baicalein reduced chemo- and radio-resistance, induced apoptosis, and suppressed stem cell-like properties in MDA-MB-231/IR cells; baicalein also reversed the expression of interferon-induced protein with tetratricopeptide repeats 2 (IFIT2), which is involved in cancer metastasis and recurrence. However, further studies are needed to evaluate the mechanisms of IFIT2 in resistant breast cancer cells [49].

Table 1 provides a detailed overview of the specific mechanisms through which flavonoids enhance radiotherapy.

\section{Anti-cancer chemotherapy resistance}

After the accidental discovery of the first DNA alkylating agent in the 1940s, several chemotherapeutic modalities were developed, becoming the first revolutionary anti-cancer pharmacological approach [9]. These include alkylating agents (the triazene compounds dacarbazine and temozolomide and the metal salts cisplatin, carboplatin, and oxaliplatin), antimetabolites (the folate analogs aminopterin and methotrexate, the purine analog mercaptopurine, and the pyrimidine analogs 5-fluorouracil, gemcitabine, and capecitabine), antimitotics (vincristine, the topoisomerase I inhibitors topotecan and irinotecan, and the microtubule-stabilizing molecules paclitaxel, docetaxel, and cabazitaxel), cytotoxic antibiotics and related substances (daunomycin/daunorubicin, actinomycin $\mathrm{D}$, and doxorubicin), polyamine inhibitors and ironmodulating drugs (ciclopirox and triapine), and combination chemotherapy regimens [9]. Chemotherapeutic agents target cancer cells and all rapidly dividing cells [14] and are often associated with primary or acquired resistance [9].
Cancer cells gradually develop resistance to almost all chemotherapeutics through various mechanisms. Cancer drug resistance is associated with increased drug efflux, alterations in drug metabolism, transport, and signal transduction molecules, elevated DNA repair capacity and apoptotic evasion, increased mutations, reactivation of drug targets, crosstalk with the cancer microenvironment and cancer cell-stroma interactions, epithelial-mesenchymal transition (EMT)-mediated chemoresistance, epigenetic mechanisms, metabolic alteration, and the effects of CSCs $[16,90]$. Despite initial responses to therapy due to the majority of cells being sensitive to the drug, the pre-existence of resistant cell subpopulations can result in relapse after chemotherapy. Resistant CSCs are involved in chemotherapy resistance in various cancer types. Intrinsic resistance can be mistaken with acquired, as resistance seems to be acquired due to therapy [17]. Both resistance factors interact and jointly modulate drug resistance. Indeed, $90 \%$ of cancer progression during and after chemotherapy is associated with drug resistance [90]. Prolonged administration of a chemotherapeutic agent can result in resistance to multiple other structurally unrelated agents, a phenomenon known as multidrug resistance (MDR) [16]. Therefore, it is necessary to provide new strategies to overcome cancer cells' resistance to chemotherapeutic agents [90]. Figure 3 provides a detailed overview of specific mechanisms related to cancer cell drug resistance. One key mechanism involves increased drug efflux associated with the overexpression of aldehyde dehydrogenase (ALDH) and the ATP-binding cassette $(A B C)$ transporter family of proteins. This is associated with drug resistance, increased DNA repair capacity and tolerance to DNA damage, genetic factors such as abnormal activation of the androgen receptor (AR) signalling pathway, PI3K/Akt signalling, epigenetic factors, increased xenobiotic metabolism, and other mechanisms including contributions from endoplasmatic reticulum (ER) stress, the receptor for advanced glycation end products (RAGE), NF-kB, and galectin-3.

Current research highlights the potential of co-administration of natural compounds such as flavonoids with chemotherapeutic agents as an attractive strategy to overcome chemotherapeutic resistance and MDR in tumors [105].

\section{Flavonoids enhance effectiveness of conventional chemotherapeutic agents}

The resistance or insensitivity of cancer cells to chemotherapeutics is a serious disadvantage of cytotoxic anti-cancer therapies. However, current research highlights the potential importance of flavonoids in increasing the sensitivity and/or efficacy of chemotherapeutic agents.

Several flavonols, including quercetin, kaempferol, or morin, exert potent capacities to modulate cancer cell 


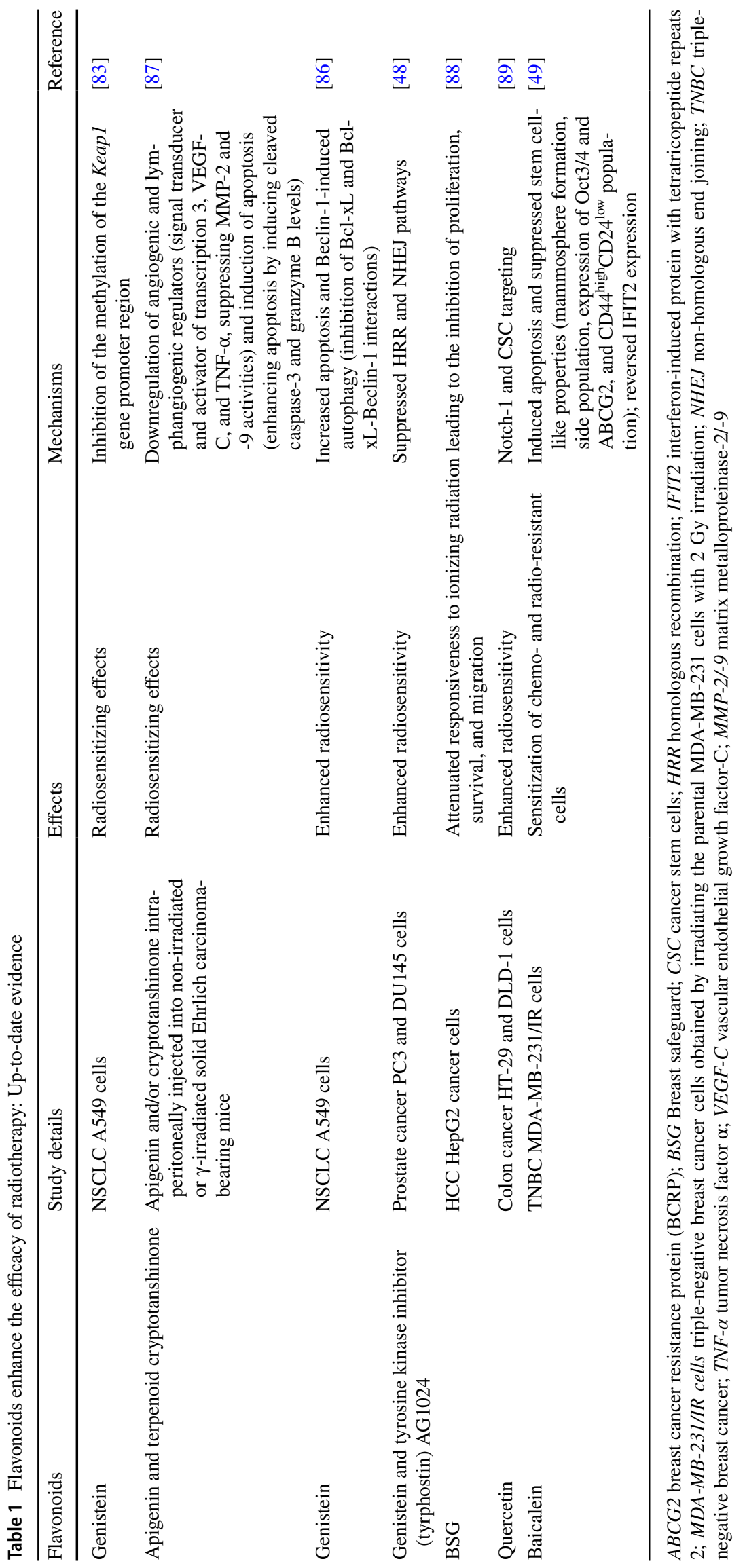


chemoresistance [50-53, 98, 103, 106, 107]. Abnormal activation of $A R$ and PI3K/Akt signaling is considered a significant cause of docetaxel resistance. Interestingly, quercetin revealed docetaxel resistance-reversing effects in docetaxelresistant prostate cancer (LNCaP/R, PC-3/R) cells in vitro and in a prostate cancer xenograft model in vivo by reversing the upregulation of P-gp, the development of mesenchymal and stem-like cell phenotypes, and the activation of androgen receptor and PI3K/Akt signaling pathways; moreover, the combinatory treatment of quercetin and docetaxel slowed tumor growth and robustly inhibited proliferation in vivo [98]. Similarly, quercetin enhanced the therapeutic efficiency of paclitaxel in prostate cancer PC-3 cells in vitro through the induction of ER stress and ROS production; this combinatory treatment also exerted beneficial effects in a PC-3 cancer-bearing murine model in vivo [107]. Also, quercetin promoted cell death and gemcitabine sensitivity in human pancreatic cancer MIA Paca-2 and MIA Paca-2 (GEMresistant) cells through the receptor for advanced glycation end products (RAGE)/PI3K/AKT/mTOR axis, especially through RAGE inhibition [103]. Quercetin also enhanced doxorubicin, paclitaxel, and vincristine activity and thus reversed MDR in breast cancer MCF-7 and doxorubicinresistant MCF-7 (MCF-7/Dox) cells by downregulating P-gp and eliminating CSCs through YB-1 nuclear translocation [50].

Kaempferol combined with 5-FU exerted a synergistic inhibitory effect on cell viability, enhanced apoptosis, and induced cell cycle arrest in both chemo-resistant and sensitive colon cancer LS174 cells. Kaempferol also blocked the production of reactive oxygen species (ROS) and modulated the expression of JAK/STAT3, MAPK, PI3K/AKT, and NF- $\mathrm{\kappa B}$ signaling in these cells [52]. As discussed above, MDR, a state of certain cancers becoming cross-resistant to structurally diverse antineoplastic agents, is associated with the overexpression of $\mathrm{ABC}$ transporters [108]. However, kaempferol exerted an ability to inhibit MDR by downregulating $\mathrm{ABCB} 1, \mathrm{ABCC} 1, \mathrm{Akt}$, and BCL2 in leukemia HL-60 and NB4 cells [53].

Co-treatment with morin and cisplatin led to the synergistic sensitization of ovarian cancer SK-OV-3 (cisplatinresistant) cells to cisplatin. Further, the sensitization of ovarian cancer cells to cisplatin is suggested to be achieved through the downregulation of galectin-3 (essential for various cellular processes such as apoptosis) by morin [106]. Moreover, morin hydrate reversed the acquired resistance of cisplatin-resistant hepatocellular cancer HepG2 ${ }^{\mathrm{DR}}$ cells by impairing PARP-1/HMGB1-dependent autophagy; indeed, PARP-1 autophagy appears to be regulated by the PARylation of HMGB1 [51].

In addition to flavonols, the flavanone hesperetin sensitized cisplatin (DDP)-resistant human lung cancer cells (A549/DDP) to cisplatin in vivo and in vitro, mechanistically through decreased expression of P-gp and increased intracellular accumulation of the P-gp substrate, rhodamine 123 [109]. Similarly, poncirin, a flavanone glycoside with a bitter taste, enhanced sensitivity to cisplatin by decreasing the expression of MDR-1, MRP1, and BCRP and inhibiting PI3K/Akt signaling in cisplatin-resistant osteosarcoma (OS) cells [110].

Moreover, other classes of flavonoids such as chalcones also exhibit potent chemosensitizing capacities in cancer models. The combination of xanthohumol, a prenylated flavonoid from hops, and the chemotherapeutic agent SN38, the active metabolite of irinotecan, in resistant colon cancer SW480 cells decreased cell viability compared with SN38 alone. Therefore, xanthohumol can be potentially utilized as a chemosensitizer of SN38 [111]. Another chalcone, flavokawain-B, showed potent anti-cancer abilities in gemcitabine-resistant NSCLC cells by inducing apoptosis and ROS production and blocking the PI3K/Akt signalling pathway [112].

Furthermore, Fan et al. recently evaluated the inhibitory effects of flavonoids on breast cancer resistance protein (BCRP) in vitro and in vivo. Eleven flavonoids (amentoflavone, apigenin, biochanin A, chrysin, diosmin, genkwanin, hypericin, kaempferol, kaempferide, licochalcone A, and naringenin) significantly inhibited BCRP in BCRPoverexpressing (BCRP-MDCKII) cells. Simultaneously, these effects were associated with reduced BCRP-mediated doxorubicin and temozolomide efflux and increases in the drugs' cytotoxicity. Also, mitoxantrone's co-administration with the above-mentioned flavonoids promoted the $\mathrm{AUC}_{0-\mathrm{t}}$ of mitoxantrone to different extents in a Sprague-Dawley rat model [113].

Moreover, dihydromyricetin, a natural flavonoid from the leaves of Vitis heyneana, reversed MRP2-induced MDR by preventing NF-kB-Nrf2 signaling in colorectal cancer HCT116/OXA and HCT8/VCR cell lines [114]. Also, bavachinin, tephrosin, and candidone sensitized MDR MCF7/ MX and EPG85.257RDB cells to daunorubicin and mitoxantrone [115].

Table 2 provides a detailed overview of specific mechanisms through which flavonoids enhance the therapeutic efficacy of conventional chemotherapeutic agents. These results suggest a significant potential of increased therapeutic efficacy through a combination of flavonoids and conventional chemotherapeutic agents.

\section{Nanotechnologic approch to facilitate flavonoid-conducted chemotherapeutic anti-cancer toxicity}

Although flavonoids demonstrate significant anti-cancer and chemosensitizing efficacy in preclinical research, their poor solubility and bioavailability are associated with 


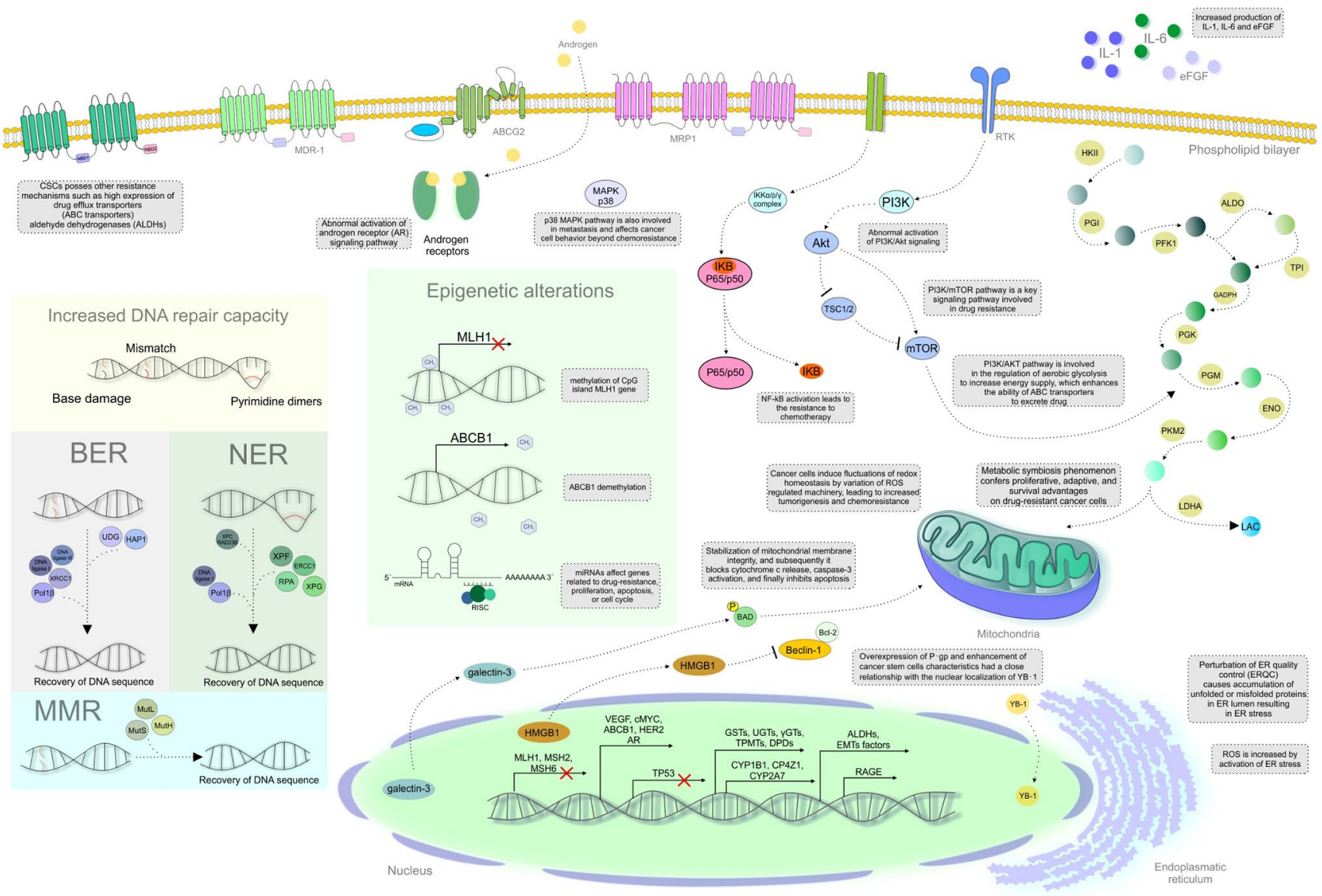

lesser effectiveness in vivo. However, as discussed below, current research highlights the potential enhancement of flavonoid-chemotherapy interaction through nanotechnology. Khonkarn et al. demonstrated that polymeric micelles of benzoylated methoxy-poly (ethylene glycol)-b-oligo( $\varepsilon$ caprolactone) or mPEG-b-OCL-Bz loaded with quercetin could represent an attractive tool to overcome MDR in cancer cells. Eventually, the combination of polymeric micelles (inhibiting P-gp efflux) and quercetin (interfering with the mitochondrial membrane potential) may represent crucial factors for the reversal of MDR in K562/ADR cells; quercetin also enhanced the cytotoxicity of doxorubicin and daunorubicin [116]. Also, the co-encapsulation of paclitaxel and naringin in mixed polymeric micelles improved the intracellular uptake and in vitro cytotoxicity of paclitaxel against breast cancer cells [117]. Further, the double-targeted nanocarrier, Quercetin-3'3-dithiodipropionic acid-Astragalus polysaccharides-Folic acid (QDAF), was synthesized and selfassembled into a neoteric nano-targeted delivery strategy, named nano-pomegranates, to effectively suppress MDR in estrogen receptor $\alpha(E R \alpha)$-positive breast cancer. Indeed, nano-pomegranates enhanced cellular uptake, apoptosis, and necrosis in MCF-7 cells in vitro and showed improved anticancer efficacy and lower systemic toxicity in vivo [118].
In conclusion, the co-delivery of conventional chemotherapeutic agents with flavonoids in nanocarrier systems could improve chemotherapeutics' efficacy and enhance the chemosensitivity of cancer cells, inhibit chemoresistance, and reduce the cytotoxicity of chemotherapeutics in healthy tissues.

\section{Resistance to anti-cancer treatments}

Conventional chemotherapy often fails due to resistance. Therefore, it was necessary to develop new strategies to improve the individual therapeutic efficacy of specific cancer types [119]. Molecular biology offers ideas for the development of selective drugs specifically targeted against certain tumors [9]. Similar to traditional chemotherapy, targeted anti-cancer agents modulate specific cellular processes (such as growth inhibition, apoptotic induction, and metastatic restriction). Unlike traditional chemotherapy, targeted cancer therapy also targets unique molecular changes associated with specific cancer types [14]. Thus, targeted cancer therapies focus on mutant proteins and signalling pathways essential for cancer cell survival and progression [120]. The term "targeted therapy" describes all treatment approaches affecting specific 
4Fig. 3 Mechanisms of resistance to chemotherapeutic drugs. Explanatory notes: A) increased drug efflux - proteins in the ATP-binding cassette $(\mathrm{ABC})$ transporter family contain nucleotide-binding domains (NBD) and two transmembrane domains (TMDs); ATP hydrolysis-driven conformational changes of TMD result in unidirectional transport across the lipid bilayer [91]. ABC transporter overexpression is observed in several cancer types and is more predominant in cancer stem cells (CSCs) [92]. ABC transporters, including multidrug resistance protein 1 (MDR-1, ABCB1, P-gp), multidrug resistance-associated protein 1 (MRP1, $\mathrm{ABCC} 1$ ), and breast cancer resistance protein (BCRP, ABCG2), are implicated in drug-resistant cancers [93]. Also, aldehyde dehydrogenase (ALDH) promotes drug resistance. $\mathrm{ABC}$ transporters and $\mathrm{ALDH}$ are upregulated in normal stem cells, CSCs, and drug-resistant cancer cells [94]. B) Increased DNA repair capacity [95] or tolerance of DNA damage [96] induced by therapeutic agents $[95,96]$-base excision repair (BER) involves different proteins (UDG, HAP1, Pol $\beta$, XRCC1, and DNA ligase I or III) [96] and nucleotide excision repair (NER) mechanisms involve damage recognition/excision proteins and helicase proteins (DNA damage is recognized by the NER protein XPC-RAD23B, which binds to DNA strand, an oligonucleotide containing the lesion is then excised from the DNA strand, a repair patch is synthesized, and DNA ligases join the patch to the DNA) [96]. NER-induced resistance to platinum-based agents $[12,96]$ includes the DNA repair endonuclease $\mathrm{XPF}$ and the DNA excision repair protein ERCC1. Replication protein A (RPA) is involved in the DNA-damage response (DDR), HR, and NER [12]. Decreased mismatch repair (MMR) promotes damage tolerance and enhanced mutagenicity and chemoresistance in cancer cells (hypermethylation of the hMLH1 gene promoter results in decreased expression of the MLH1 protein involved in the MMR pathway) [12]. C) Genetic and epigenetic factors-TP53 loss results in continued replication and resistance to genotoxic drugs [12]. Abnormal activation of the androgen receptor (AR) signaling pathway (AR over-expression, AR gene amplification, mutations, alterations in coregulators, and continuous androgen release from the tumor tissue or adrenal glands) and abnormal activation of PI3K/ Akt or PI3K/Akt/mTOR signaling can lead to the overexpression of $\mathrm{ABC}$ transporters and the upregulation of oncogenes and growth factors such as VEGF and c-myc. The acidified tumor micro-environment promotes aerobic glycolysis and MDR (by reducing drug absorption and efficiency). PI3K/Akt regulates aerobic glycolysis to increase energy supply and enhance $\mathrm{ABC}$ transporter-mediated drug excretion [97]. The transcription of specific genes essential for resistance is enhanced (e.g. $A B C B$ amplification) [12, 98, 99]. Epigenetic alterations (genome-wide DNA hypomethylation, regional hypermethylation, changes in histone modifications, and alterations in miRNA expression) [12] - carboplatin-induced methylation of the $M L H 1 \mathrm{CpG}$ island (important for the MMR DNA repair system) is associated with chemoresistance in ovarian cancer; $A B C B 1$ demethylation decreases the accumulation of anti-cancer drugs and promotes the acquisition of the multidrug phenotype [12]. D) Growth factors-cytokine (IL-1, IL-6) production is increased in multidrug cancer cells when compared with drug-sensitive cancer cells [12]. Specific chemotherapeutic agents were ineffective against cancers with increased levels of extracellular fibroblast growth factors (eFGF) [12]. E) Increased metabolism of xenobiotics-altered expression of isoforms of cytochrome (CYPs)—overexpressed CYP1B1, CP4Z1, CYP1B1, and CYP2A7 and phase II enzymes, such as glutathioneS-transferases (GSTs), uridine diphospho-glucuronosyltransferases (UGTs), gamma-glutamyl transferases $(\gamma \mathrm{GTs})$, thiopurine methyltransferases (TPMTs), and dihydropyrimidine dehydrogenases (DPDs) promote the development of multidrug resistance (MDR) [12]. F) CSCs - targeted less by chemotherapeutic drugs (due to slow cell cycle kinetics, high expression of ABC transporters, ALDHs, epithelial-mesenchymal transition, and factors affecting the tumor microenvironment, such as hypoxia, and epigenetic modifications)
[100]. F) Other mechanisms include endoplasmatic reticulum (ER) stress - perturbation of ER quality control (ERQC) causes the accumulation of unfolded or misfolded proteins in the ER lumen, resulting in ER stress. The ER stress response (ERSR) is produced to restore homeostasis or activate cell death. ERS is critical for chemo-therapeutic resistance, following the initiation of an ERSR [101]. ROS is increased by the activation of ER stress. Cancer cells induce fluctuations of redox homeostasis through the variation of ROS-regulated machinery, leading to increased tumorigenesis and chemoresistance [102]. The receptor for advanced glycation end products (RAGE) activation leads to drug resistance (pancreatic cancer) [103]. P-gp overexpression and CSCs are closely associated with the nuclear localization of $\boldsymbol{Y B}-\mathbf{1}$ in cancer cells [50]. $\boldsymbol{N F - k B}$ activation rescues cancer cells from cell death [104]. Galectin-3 is transported from the nucleus to the cytoplasm to stimulate the phosphorylation of $\mathrm{Bcl}-2$ associated death (Bad) protein and the downregulation of Bad; this results in the maintenance of mitochondrial membrane integrity. Consequent effects, including the blockade of cytochrome c release and caspase- 3 activation, inhibition of apoptosis, and activation of $\boldsymbol{P A R P 1}$, induce chemoresistance through the cytosolic translocation of HMGB1 via PARylation, which is known to induce autophagy by disrupting the interaction between Beclin-1 and Bcl-2 [51]

molecular targets and involves small selective inhibitory molecules and biological drugs such as monoclonal antibodies targeted against specific cellular receptors and proteins of neoplastic processes. Examples of monoclonal antibodies include bevacizumab, cetuximab, pertuzumab, and trastuzumab [9]. The targets of selective tyrosine kinase and serine/threonine-protein kinase small molecule inhibitors include growth factors, cell-cycle proteins, apoptotic modulators, signalling molecules, and molecules promoting angiogenesis. Imatinib, dasatinib, and nilotinib are selective tyrosine kinase inhibitors (TKIs). Small molecules targeting tyrosine kinase proteins include gefitinib and erlotinib. Further, lapatinib is a potent HER 1/2 inhibitor. VEGF inhibitors are another class of TKIs, including sunitinib and sorafenib. Another class of selective small molecules includes mTOR inhibitors (temsirolimus and everolimus), BRAF inhibitors (vemurafenib and dabrafenib), MEK inhibitors (trametinib and cobimetinib), and inhibitors of proteasome machinery (bortezomib) [9]. In addition, abivertinib is a novel third generation epidermal growth factor receptor (EGFR) TKI [121] that inhibits Bruton's tyrosine kinase (BTK), which exerts an oncogenic role in the proliferation and survival of many B cell malignancies [122]. Figure 4 provides an overview of mechanisms related to the resistance of cancer cells to targeted anti-cancer agents.

The resistance to single-agent targeted therapy is related to the occurrence of many cancer mutations, making such tumors less dependent on a single oncogenic event and more reliant on dynamic interconnected signalling pathways and tumor heterogeneity, especially in an advanced and metastatic stage [126]. Compared with the mode of resistance to cytotoxic agents associated with deregulated pharmacokinetics such as drug efflux, resistance to 


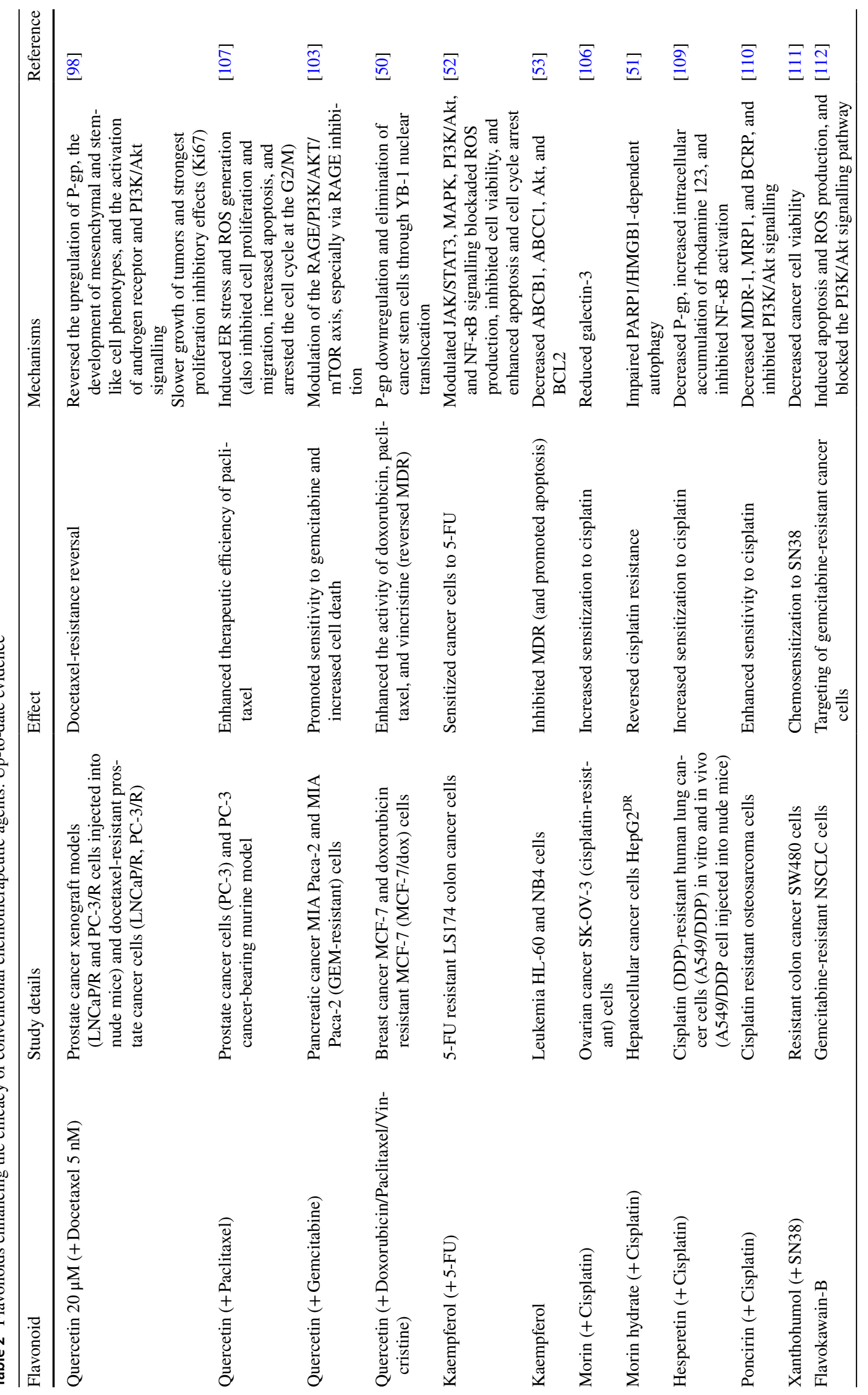


targeted therapy is usually a result of target gene mutations or the activation of pro-survival signaling. Therefore, combination therapy with next-generation agents, such as flavonoids, could target mutations and pathways associated with resistance as part of a personalized approach to mitigate targeted drug resistance in cancer patients [127].

\section{Flavonoids enhance effectiveness of targeted anti-cancer therapy}

Targeted therapy tremendously enhances cancer management; however, acquired and intrinsic resistance are major limitations of targeted anti-cancer treatment [126]. Nevertheless, as discussed below, current research highlights the potential importance of flavonoids in increasing the sensitivity to and/or efficacy of current targeted anti-cancer agents.

Trastuzumab is a recombinant humanized monoclonal antibody targeted against the human epidermal growth factor receptor 2 (HER2) tyrosine kinase receptor and is used for the treatment of HER2-positive breast cancer. The anthocyanins cyanidin-3-glucoside and peonidin-3-glucoside inhibited trastuzumab-resistant breast cancer MDA-MB-453R and BT474R cells in vitro and in a murine xenograft model in vivo [128].

TKIs are novel target-specific anti-cancer drugs. Nevertheless, the disadvantage of TKIs usage is the development of resistance [54]. The existence of EGFR mutations in NSCLC led to changes in the traditional lung cancer regimen from traditional cytotoxic chemotherapy to molecularly targeted agents. Superior to traditional chemotherapy, EGFR TKIs are considered a standard first-line treatment modality for advanced NSCLC [129]. However, patients receiving EGFR TKIs usually develop resistance. Hydroxygenkwanin HGK, a novel flavonoid, exerted potent antitumor activity against TKI-resistant NSCLC cells by promoting the degradation of EGFR [55]. Similarly, the combination of apigenin and gefitinib [56], an orally active anilinoquinazoline that selectively and reversibly inhibits intracellular EGFR TKIs activity [130], could represent a strategy for acquired resistance to EGFR-TKIs in NSCLS as it blocked autophagy flux and induced apoptosis in lung cancer EGFR L858R-T790M-mutated H1975 cells [56]. The flavone apigenin synergized with abivertinib [122], a novel third-generation EGFR TKI [121] targeting BTK, inhibits diffuse large B-cell lymphoma in vitro (U2932, LY10, OCI-LY10 cells) and in a murine xenograft model through the inhibition of $\mathrm{p}-\mathrm{GS} 3 \mathrm{~K}-\beta$ and its downstream targets; therefore, the ability of apigenin to synergize with BTK inhibitors is important for the improvement of targeted therapy, especially to overcome developed resistance [122]. Moreover, resistance mediated by BCR-ABL limits the utilization of TKIs in leukemia. Nevertheless, the chalcone xanthohumol attenuated the autophagy induced 


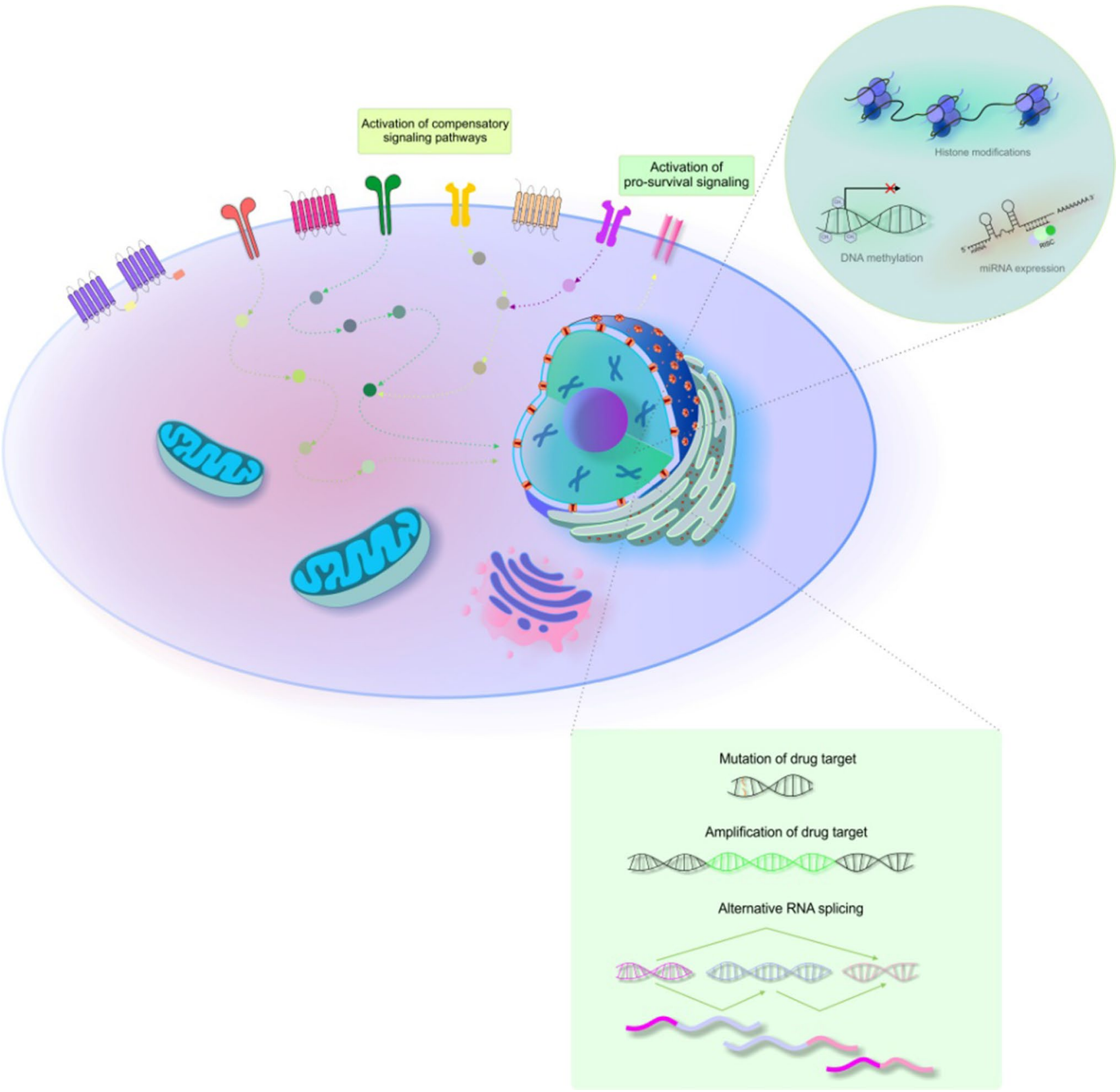

Fig. 4 Mechanisms of cancer cells resistance to targeted therapy. Explanatory notes: Resistance to targeted therapy often results from reactivation of pathways inhibited by the drug (acquisition of drugresistant mutations/amplification of the target, re-activation of downstream signalling proteins via activation mechanisms or activating mutations, or activation of compensatory signalling pathways) [123]. Due to commonly observed gene mutations, cancer cells can perform modifications as a response to targeted molecules and thus induce resistance to specific agents [12]. The mutation, amplification, down-

by imatinib, a small molecule TKI used to treat chronic myelogenous leukemia and enhanced its therapeutic efficacy in myelogenous leukemia K562 cells [111]. Further, Trifolium flavonoids showed a capacity to overcome resistance to gefitinib, EGFR-TKI, through suppressing ERK and regulation, and alternative RNA splicing of drug targets all contribute to the resistance of cancer cells to targeted therapy [124]. Moreover, direct restoration of biologic function that was disrupted by a drug [125], activation of compensatory pathways parallel to or downstream of the inhibited pathway (such as pro-angiogenic signalling through PDGFR), activation of pro-survival signalling, and epigenetic alterations (like DNA methylation, histone modifications, and microRNA) also contribute to resistance to targeted treatment [124]

STAT3 signaling in NSCLC cell line PC-9R [131]. A novel EGCG (flavonol) derivative isolated from Anhua dark tea sensitized NSCLC gefitinib-resistant cells HCC827-Gef to gefitinib through the suppression of $\mathrm{PI} 3 \mathrm{~K} / \mathrm{mTOR}$ signalling and EMT [132]. 
Sorafenib is a multikinase angiogenesis inhibitor [9] used as first-line therapy in HCC. However, patients who initially benefit from sorafenib usually develop resistance within 6 months [133]. A proposed mechanism of this resistance is the expression of the pregnane $X$ receptor (PXR) or MDR-1, which is related to the elimination of sorafenib in HCC cells. Interestingly, the flavone rhamnetin is an inhibitor of sirtuin (Sirt) 1 and could inhibit the downstream (PXR) gene-MDR-1, via the miR-148a/PXR axis. Therefore, rhamentin decelerated the metabolic clearance of sorafenib and also sensitized HCC cells to the drug [134]. Similarly, the combinatory treatment with apigenin potentiated the cytotoxicity of sorafenib in HCC HepG2 cells, as demonstrated through decreased cell viability, decreased migration and invasion, and increased apoptosis compared with single treatment groups [135]. Also, Saraswati et al. demonstrated the capability of a chalcone phloretin to overcome sorafenib resistance in HCC as demonstrated through Src homology region 2 domain-containing phosphatase-1 (SHP-1)-mediated inhibition of STAT3 and Akt/VEGFR2 [136]. As stated above, the disadvantage of TKIs usage is resistance; important mechanisms of the development of resistance include enhanced TKI efflux through efflux transporters such as BCRP [54]. 5,7-dimethoxyflavone effectively inhibited BCRP-1-mediated sorafenib efflux in Madin-Darby Canine Kidney Type II wild-type cell subclones that were transfected with murine Bcrp1 (MDCK/Bcrp1); these results highlight an essential potential of 5,7-dimethoxyflavone as a chemosensitizing agent in BCRP-mediated drug resistance [54]. Further, the flavonol kaempferol enhanced the chemotherapeutic efficacy of sorafenib against HCC demonstrated in silico and in vitro (liver cancer HepG2 and N1S1 cells); also, kaempferol reversed MDR by decreasing P-gp overexpression [137].

Moreover, the flavonoid derivative WYC0209 is a potential adjuvant agent against CD133-driven urothelial carcinoma (UC) CSCs and could serve as a potent strategy against UC therapeutic resistance; among others, WYC0209 declined EMT-CSCs markers such as MDR-1 or ABCG2 in vitro [138].

Furthermore, the flavone scutellarin potentiated the activity of bortezomib (a proteasome inhibitor), circumvented chemoresistance, promoted apoptosis, and repressed tumor growth in a murine xenograft model of multiple myeloma through the HDAC/miR-34a-mediated down-modulation of $\mathrm{Akt} / \mathrm{mTOR}$ and NF- $\mathrm{KB}$ signaling [139].

Also, histone deacetylase inhibitors (HDACi) and tumor necrosis factor-associated apoptosis-inducing ligand (TRAIL) represent other targeted anti-cancer therapeutic strategies [140, 141]. HDACi is a novel class of smallmolecular therapeutics that target the regulation of histone and non-histone proteins [142]. The flavonol fisetin is a potential complementary agent in HDACi resistance, as it improves the chemosensitivity of HA22T, apicidin-resistant, and suberoylanilide hydroxamic acid-resistant (SAHAR) HCC cells. Fisetin synergistically interacted with HDACi in parental cells and also resistant cell lines. Fisetin also promoted therapeutic potential in the xenograft model generated from HDAC inhibitor-resistant cells [141]. Further, TRAIL is an immune cytokine of the TNF family that received attention as a targeted anti-cancer agent through the selective induction of apoptosis in cancer cells [143, 144]. Despite tumor TRAIL's potential as a potent anticancer agent inducing apoptosis of cancer but not normal cells, colon cancer is often TRAIL-resistant. Mutations in DR4 and DR5, domains of death receptors associated with TRAIL-induced apoptosis, induce cancer cell resistance to TRAIL. However, icariin (a prenylated flavonol glycoside derived from Epimedium sagittatum) sensitized HCT116 colon cancer cells to TRAIL-induced apoptosis through the upregulation of DR5 and DR4 (mediated by ROS, ERK, and transcription factor CCAAT enhancer-binding protein homologous protein/CHOP/) in vitro and in vivo (xenograft mouse model) [140].

Table 3 provides a summary of the mechanisms through which flavonoids enhance the therapeutic efficacy of targeted anti-cancer agents.

\section{Nanotechnological and combinatorial approaches enhance effectiveness of the flavonoids-conducted therapy}

Combinatorial and nanoparticulate approaches are suggested to overcome the challenges of resistance and severe side effects posed by monotherapies. Currently, the combinatory therapy of a chemotherapeutic agent and phytochemicals or chemotherapy and targeted therapy is an important tool to improved cancer patient management. Chemotherapy combined with targeted therapy is suggested to be effective especially for advanced NSCLC while EGFR is an essential target in NSCLC patients. Cetuximab, a monoclonal antibody targeting EGFR, is a first-line treatment for NSCLC, advanced colorectal cancer, and head and neck cancers. Indeed, cetuximab-functionalized nanostructured lipid carriers were developed for the co-delivery of paclitaxel and 5-demethylnobiletin (a hydroxylated polymethoxyflavone from citrus) and to avert dose-related adverse effects of anti-cancer agents. These nanostructured lipid carriers effectively inhibited tumor growth in a model of A549 paclitaxel-resistant cell-bearing mice [145].

In conclusion, flavonoids represent an effective tool to improve the therapeutic outcomes of targeted anti-cancer strategies facing evident disadvantages such as insensitivity and resistance. 


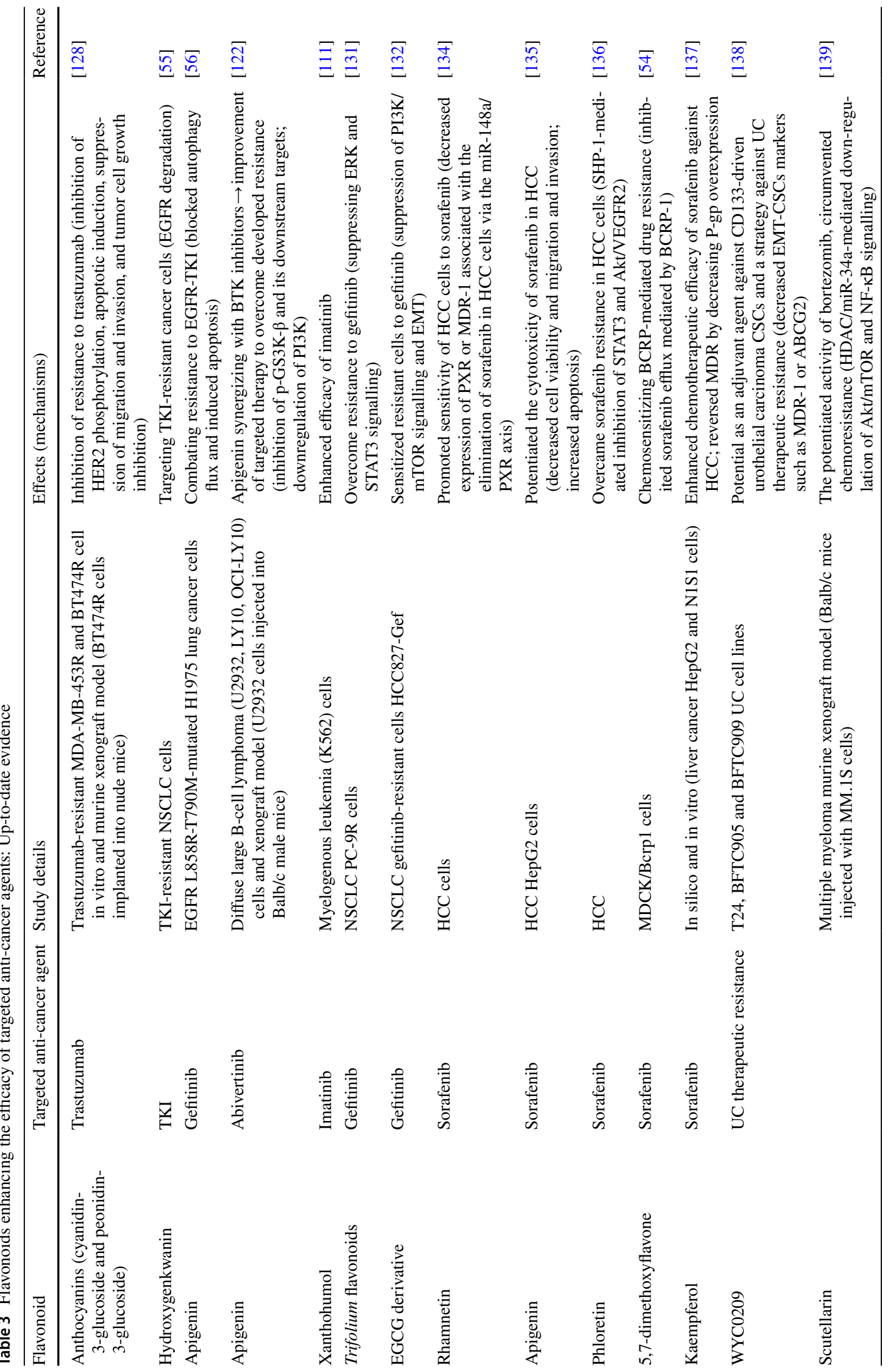




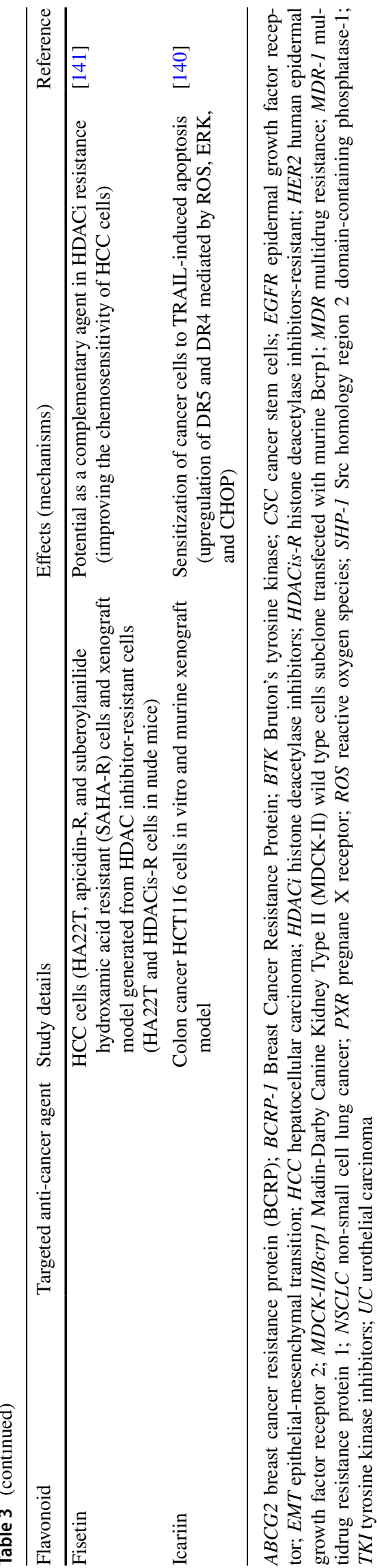

\section{Anti-cancer immunotherapy}

\section{Imuunotherapy revolutionized treatments of malignancies}

After 2010, cancer immunotherapy research introduced new monoclonal antibodies targeting tumor antigens and T-cell protein receptors to downregulate the immune response, specifically the immune checkpoint inhibitor monoclonal antibodies anti-cytotoxic T-lymphocyte-associated antigen 4 (anti-CTLA4) and anti-programmed cell death protein 1 antibody (anti-PD1). Monoclonal antibodies directed against immune checkpoint inhibitors include ipilimumab, nivolumab, and pembrolizumab [9]. Immunotherapy is a promising tool for cancer management, as it restores the anti-tumor immune response [15]. Not all patients respond to immunotherapy; thus, it is necessary to improve its efficacy [15].

Some level of immune escape and resistance is intrinsic to malignancies due to the development of most human tumors in an immune-competent environment. However, acquired resistance to immunotherapy can result from pre-existing genetic and epigenetic traits or de novo alterations of cancer cells or other tumor microenvironmental components. Thus, cancer cells can evade the immune response intrinsically (loss or downregulation of target antigen expression, defective antigen presentation, insensitivity to immune effector molecules, upregulation of alternative immune checkpoints, and epigenetic alterations) or via extrinsic mechanisms, which are mediated by non-cancer cells of the tumor microenvironment including tumor-associated macrophages (TAMs), regulatory T cells (Tregs), and myeloidderived suppressor cells (MDSCs) [146].

Programmed death ligand 1 (PD-L1) is an essential immune checkpoint protein that binds to programmed death 1 (PD-1) on $\mathrm{T}$ lymphocytes. Indeed, $\mathrm{T}$ cells exert an essential role in the eradication of cancer cells. However, cancer cells escape the immune response through PD-L1 expression. The binding of PD-L1 to PD-1 results in the inhibition of T-cell proliferation and activity, leading to tumor immunosuppression [147]. Although PD-L1/PD-1 checkpoint inhibition revolutionized the treatment of various malignancies, such therapy is still ineffective in a significant percentage of patients due to primary or acquired resistance [148].

Due to the ineffectiveness of immunotherapy and the experience of resistance in some cases, the antitumor efficacy of cancer immunotherapy needs to be increased. Thus, immunotherapeutic agents are often administered in combination with each other or with chemotherapeutic agents, radiotherapy, or surgery. Also, the combination of immunotherapy with antiangiogenic drugs yields promising outcomes [9, 149]. It is also essential to emphasize the potential of phytochemicals and their derivatives to improve cancer immunotherapy responses in the development of novel immunotherapeutic strategies $[150,151]$. 


\section{Flavonoids enhance effectiveness of anti-cancer immunotherapy}

As discussed below, the anti-cancer effects of flavonoids are also applicable in cancer immunotherapy either in combination with other agents or single agents [57, 59, 152-155].

Due to the frequent development of resistance to sorafenib, the first-line therapy for HCC, immune checkpoint inhibitors (ICI) such as nivolumab are studied as alternatives. However, due to the often unsuccessful outcomes of immunotherapy, the combinatorial approach seems to be a better choice to improve the treatment and to block immunosuppressive signals in the tumor microenvironment. Although the coadministration of VEGF inhibitors and ICI is associated with synergistic anti-cancer effects, it exerts several adverse effects. However, phytochemicals including flavonoids could improve the plant-based antiangiogenic-immunotherapy combination in $\mathrm{HCC}$ when compared with single compounds that are often associated with therapeutic failure [152].

Furthermore, flavopiridol is a synthetic flavonoid that inhibits cyclin-dependent kinases [156]. Although most chronic lymphocytic leukemia (CLL) patients receiving chemoimmunotherapy achieve complete remission, patients with significantly shortened progression-free intervals still represent an important obstacle. Also, minimal residual disease (MRD) occurs in a majority of CLL patients who relapse. Moreover, a phase I clinical trial demonstrated flavopiridol to be safe and efficient as consolidation therapy after chemoimmunotherapy in CLL patients [153].

As discussed above, the immune escape of cancer cells is associated with PD-L1 expression [147]. Also, the chemoresistance of nasopharyngeal carcinoma is associated with the upregulation of checkpoint inhibitor PD-L1, which is linked to enhanced aerobic glycolysis promoted by HIF1- $\alpha$ deregulation and LDH-A activity. However, silibinin downregulated PD-L1 expression by modulating HIF-1 $\alpha / \mathrm{LDH}-\mathrm{A}$-mediated metabolism in nasopharyngeal carcinoma C666-1 cells and thus provided a potential avenue to overcome PD-L1-mediated resistance [57].

Moreover, checkpoint blockade is an effective treatment of lung cancer; however, it often leads to resistance. Therefore, Tang et al. aimed to develop a new strategy to improve checkpoint blockade therapy. Eventually, dual inhibition of COX-2 and EGFR by melafolone improved PD-1 immunotherapy against Lewis lung carcinoma and CMT167 tumors; these results highlight its important role as a combinatory strategy against lung cancer by affecting vessels and immune cells [59]. Further, the prenylated flavonoid icaritin exerts potent anti-cancer activity by modulating multiple biochemical and cellular responses [58]. Advanced HCC is associated with limited treatment options. It is suggested that icaritin has the potential as an oral immunotherapeutic agent used alongside immune-checkpoint inhibitors (antibody-based PD-1/PD-L1 blockade therapies). As the authors demonstrated in a phase I trial, the preliminary durable survival benefits of icaritin in advanced HCC patients correlated with its immuno-modulatory activities and immune biomarkers [154]. Similarly, apigenin also suppressed PD-L1 in vitro in melanoma cells and in host dendritic cells; this potentiated the cytotoxicity of cocultured cytokine-induced killer cells against melanoma cells [155].

In conclusion, flavonoids improve cancer immunotherapeutic effects either through increased efficacy of other anti-cancer agents or as potent single molecules modulating immune responses of cancer cells.

\section{Conclusions and expert recommendations in context of predictive, preventive and personalized (3P) medicine}

Cost-efficacy of currently applied treatments is an issue in overall cancer management challenging healthcare and causing tremendous economic burden to societies around the world. Consequently, complex treatment models presenting concepts of predictive diagnostics followed by the targeted prevention and treatments tailored to the individualized patient profiles earn global appreciation as benefitting the patient, healthcare economy, and the society at large.

In this context, application of flavonoids as a spectrum of compounds and their nano-technologically created derivatives is extensively under consideration, due to their multi-faceted anti-cancer effects applicable to the overall cost-effective cancer management, primary, secondary, and tertiary prevention.

Conventional anti-cancer strategies demonstrate evident deficits. Despite recent progress in anti-cancer strategies, the development of a therapy resistance remains the leading cause of the cancer-related mortality. An improved understanding of carcinogenic processes allows for the technological innovation creating more efficient therapeutic modalities. Targeted anti-cancer therapies leverage unique molecular changes associated with specific cancer types.

Anti-cancer protective application of flavonoids in the context of 3P medicine should follow principles of the evidence-based therapeutic effects, individualized prediction, targeted prevention and personalization of the treatment algorithms. To this end, application of specialized analytical approaches is strongly recommended such as liquid biopsy analysis, risk assessment tools, predictive and companion diagnostics, multi-omics and multi-parametric analysis, and application of artificial intelligence in medicine.

Authors' contribution A.L., P.K. and O.G. were responsible for the paper conception and final data presentation. The manuscript was drafted by A.L., M.S. (Marek Samec), L.K., A.B., C.B.. and critically 
revised by P.K., D.B., M.S. (Manaal Siddiqui), M.S. (Mehdi Shakibaei), M.Š. (Miroslava Šudomová), B.A, K.Z, K.B., F.A.G., and O.G.

E.K., J.D., M.P., L.K.G., S.T.S.H., and A.Z. provided skilled assistance and supervised the overall preparation of the manuscript. All authors have read and agreed to the published version of the manuscript.

Funding Open Access funding enabled and organized by Projekt DEAL. The present study was supported by the Scientific Grant Agency of the Ministry of Education, Science, Research and Sport of the Slovak Republic (Bratislava, Slovak Republic; grant no. VEGA 1/0136/19), Slovak Research and Development Agency under Contract No. APVV-16-0021 and LISPER project (grant Nr. 313011V446) in bilateral agreement with the European Association for Predictive, Preventive and Personalised Medicine.

Data availability Not applicable.

Code availability Not applicable.

\section{Declarations}

Ethics approval Not applicable.

Consent to participate Not applicable.

Consent for publication Not applicable.

Conflict of interest The authors declare no conflict of interest.

Open Access This article is licensed under a Creative Commons Attribution 4.0 International License, which permits use, sharing, adaptation, distribution and reproduction in any medium or format, as long as you give appropriate credit to the original author(s) and the source, provide a link to the Creative Commons licence, and indicate if changes were made. The images or other third party material in this article are included in the article's Creative Commons licence, unless indicated otherwise in a credit line to the material. If material is not included in the article's Creative Commons licence and your intended use is not permitted by statutory regulation or exceeds the permitted use, you will need to obtain permission directly from the copyright holder. To view a copy of this licence, visit http://creativecommons.org/licenses/by/4.0/.

\section{References}

1. Kucera R, Pecen L, Topolcan O, Dahal AR, Costigliola V, Giordano FA, et al. Prostate cancer management: long-term beliefs, epidemic developments in the early twenty-first century and 3PM dimensional solutions. EPMA Journal. 2020;11:399418. https://doi.org/10.1007/s13167-020-00214-1.

2. Koklesova L, Liskova A, Samec M, Qaradakhi T, Zulli A, Smejkal K, et al. Genoprotective activities of plant natural substances in cancer and chemopreventive strategies in the context of $3 \mathrm{P}$ medicine. EPMA Journal. 2020;11:261-87. https://doi.org/10. 1007/s13167-020-00210-5.

3. Samec M, Liskova A, Koklesova L, Samuel SM, Zhai K, Buhrmann $\mathrm{C}$, et al. Flavonoids against the Warburg phenotypeconcepts of predictive, preventive and personalised medicine to cut the Gordian knot of cancer cell metabolism. EPMA J. 2020;11:377-98. https://doi.org/10.1007/s13167-020-00217-y.
4. Liskova A, Samec M, Koklesova L, Kudela E, Kubatka P, Golubnitschaja O. Mitochondriopathies as a clue to systemic disorders-analytical tools and mitigating measures in context of predictive, preventive, and personalized (3P) Medicine. Int J Mol Sci 2021;22, https://doi.org/10.3390/ijms22042007.

5. Koklesova L, Samec M, Liskova A, Zhai K, Büsselberg D, Giordano FA, et al. Mitochondrial impairments in aetiopathology of multifactorial diseases: common origin but individual outcomes in context of 3P medicine. EPMA J. 2021;1-14, https:// doi.org/10.1007/s13167-021-00237-2.

6. Solnier J, Fladerer J-P. Flavonoids: A complementary approach to conventional therapy of COVID-19? Phytochem Rev. 2020;1-23, https://doi.org/10.1007/s11101-020-09720-6.

7. Liskova A, Samec M, Koklesova L, Samuel SM, Zhai K, AlIshaq RK, et al. Flavonoids against the SARS-CoV-2 induced inflammatory storm. Biomed Pharmacother. 2021;138:111430. https://doi.org/10.1016/j.biopha.2021.111430.

8. Crigna AT, Samec M, Koklesova L, Liskova A, Giordano FA, Kubatka P, et al. Cell-free nucleic acid patterns in disease prediction and monitoring-hype or hope? EPMA J. 2020;1-25, https:// doi.org/10.1007/s13167-020-00226-x.

9. Falzone L, Salomone S, Libra M. Evolution of cancer pharmacological treatments at the turn of the third millennium. Front Pharmacol 2018;9, https://doi.org/10.3389/fphar.2018.01300.

10. Vanneman M, Dranoff G. Combining immunotherapy and targeted therapies in cancer treatment. Nat Rev Cancer. 2012;12:237-51. https://doi.org/10.1038/nrc3237.

11. Chen HHW, Kuo MT. Improving radiotherapy in cancer treatment: Promises and challenges. Oncotarget. 2017;8:62742-58. https://doi.org/10.18632/oncotarget.18409.

12. Bukowski K, Kciuk M, Kontek R. Mechanisms of multidrug resistance in cancer chemotherapy. Int J Mol Sci 2020;21, https:// doi.org/10.3390/ijms21093233.

13. Koukourakis MI, Giatromanolaki A. Warburg effect, lactate dehydrogenase, and radio/chemo-therapy efficacy. Int J Radiat Biol. 2019;95:408-26. https://doi.org/10.1080/09553002.2018. 1490041.

14. Baudino TA. Targeted cancer therapy: the next generation of cancer treatment. Curr Drug Discov Technol. 2015;12:3-20. https:// doi.org/10.2174/1570163812666150602144310.

15. Inthagard J, Edwards J, Roseweir AK. Immunotherapy: enhancing the efficacy of this promising therapeutic in multiple cancers. Clin Sci (Lond). 2019;133:181-93. https://doi.org/10.1042/ CS20181003.

16. Pan S-T, Li Z-L, He Z-X, Qiu J-X, Zhou S-F. Molecular mechanisms for tumour resistance to chemotherapy. Clin Exp Pharmacol Physiol. 2016;43:723-37. https://doi.org/10.1111/1440-1681. 12581.

17. Wang X, Zhang H, Chen X. Drug resistance and combating drug resistance in cancer. Cancer Drug Resistance. 2019;2:141-60. https://doi.org/10.20517/cdr.2019.10.

18. Liskova A, Samec M, Koklesova L, Giordano FA, Kubatka P, Golubnitschaja O. Liquid Biopsy is Instrumental for 3PM Dimensional Solutions in Cancer Management. Journal of Clinical Medicine. Multidisciplinary Digital Publishing Institute; 2020;9:2749, https://doi.org/10.3390/jcm9092749.

19. Grech G, Zhan X, Yoo BC, Bubnov R, Hagan S, Danesi R, et al. EPMA position paper in cancer: current overview and future perspectives. EPMA J. 2015;6:9. https://doi.org/10.1186/ s13167-015-0030-6.

20. Kapinova A, Kubatka P, Liskova A, Baranenko D, Kruzliak P, Matta M, et al. Controlling metastatic cancer: the role of phytochemicals in cell signaling. J Cancer Res Clin Oncol. 2019;145:1087-109. https://doi.org/10.1007/ s00432-019-02892-5. 
21. Kapinova A, Kubatka P, Golubnitschaja O, Kello M, Zubor P, Solar P, et al. Dietary phytochemicals in breast cancer research: anticancer effects and potential utility for effective chemoprevention. Environ Health Prev Med. 2018;23:36. https://doi.org/10. 1186/s12199-018-0724-1.

22. Kubatka P, Kello M, Kajo K, Kruzliak P, Výbohová D, Šmejkal $\mathrm{K}$, et al. Young Barley Indicates Antitumor Effects in Experimental Breast Cancer In Vivo and In Vitro. Nutr Cancer. 2016;68:611-21. https://doi.org/10.1080/01635581.2016.11545 77.

23. Kubatka P, Kapinová A, Kello M, Kruzliak P, Kajo K, Výbohová D, et al. Fruit peel polyphenols demonstrate substantial anti-tumour effects in the model of breast cancer. Eur J Nutr. 2016;55:955-65. https://doi.org/10.1007/s00394-015-0910-5.

24. Kubatka P, Uramova S, Kello M, Kajo K, Samec M, Jasek K, et al. Anticancer Activities of Thymus vulgaris L. in Experimental Breast Carcinoma in Vivo and in Vitro. Int J Mol Sci. 2019;20, https://doi.org/10.3390/ijms20071749.

25. Kubatka P, Kello M, Kajo K, Samec M, Jasek K, Vybohova D, et al. Chemopreventive and Therapeutic Efficacy of Cinnamomum zeylanicum L. Bark in Experimental Breast Carcinoma: Mechanistic In Vivo and In Vitro Analyses. Molecules. 2020;25:1399. https://doi.org/10.3390/molecules25061399.

26. Kubatka P, Kello M, Kajo K, Samec M, Liskova A, Jasek K, et al. Rhus coriaria L. (Sumac) Demonstrates Oncostatic Activity in the Therapeutic and Preventive Model of Breast Carcinoma. Int J Mol Sci. 2020;22, https://doi.org/10.3390/ijms22010183.

27. Koklesova L, Liskova A, Samec M, Buhrmann C, Samuel SM, Varghese E, et al. Carotenoids in cancer apoptosis-the road from bench to bedside and back. Cancers (Basel). 2020;12, https://doi. org/10.3390/cancers12092425.

28. Kubatka P, Kello M, Kajo K, Kruzliak P, Výbohová D, Mojžiš J, et al. Oregano demonstrates distinct tumour-suppressive effects in the breast carcinoma model. Eur J Nutr. 2017;56:1303-16. https://doi.org/10.1007/s00394-016-1181-5.

29. Kubatka P, Uramova S, Kello M, Kajo K, Kruzliak P, Mojzis $\mathrm{J}$, et al. Antineoplastic effects of clove buds (Syzygium aromaticum L) in the model of breast carcinoma. J Cell Mol Med. 2017;21:2837-51. https://doi.org/10.1111/jcmm.13197.

30. Ashrafizadeh M, Ahmadi Z, Farkhondeh T, Samarghandian S. Resveratrol targeting the Wnt signaling pathway: A focus on therapeutic activities. J Cell Physiol. 2020;235:4135-45. https:// doi.org/10.1002/jcp.29327.

31. Ashrafizadeh M, Tavakol S, Ahmadi Z, Roomiani S, Mohammadinejad R, Samarghandian S. Therapeutic effects of kaempferol affecting autophagy and endoplasmic reticulum stress. Phytother Res. 2020;34:911-23. https://doi.org/10.1002/ptr.6577.

32. Ashrafizadeh M, Rafiei H, Mohammadinejad R, Afshar EG, Farkhondeh T, Samarghandian S. Potential therapeutic effects of curcumin mediated by JAK/STAT signaling pathway: A review. Phytother Res. 2020;34:1745-60. https://doi.org/10.1002/ptr. 6642 .

33. Ashrafizadeh M, Ahmadi Z, Farkhondeh T, Samarghandian S. Autophagy regulation using luteolin: new insight into its antitumor activity. Cancer Cell Int. 2020;20:537. https://doi.org/10. 1186/s12935-020-01634-9.

34. Ashrafizadeh M, Ahmadi Z, Mohammadinejad R, Ghasemipour Afshar E. Tangeretin: a mechanistic review of its pharmacological and therapeutic effects. J Basic Clin Physiol Pharmacol. 2020;31, https://doi.org/10.1515/jbcpp-2019-0191.

35. Buhrmann C, Shayan P, Brockmueller A, Shakibaei M. Resveratrol suppresses cross-talk between colorectal cancer cells and stromal cells in multicellular tumor microenvironment: a bridge between in vitro and in vivo tumor microenvironment study. Molecules. 2020;25, https://doi.org/10.3390/molecules25184292.
36. Buhrmann C, Popper B, Kunnumakkara AB, Aggarwal BB, Shakibaei M. Evidence that calebin a, a component of curcuma longa suppresses NF-B mediated proliferation, invasion and metastasis of human colorectal cancer induced by TNF- $\beta$ (Lymphotoxin). Nutrients. 2019;11, https://doi.org/10.3390/nu111 22904.

37. Bhia M, Motallebi M, Abadi B, Zarepour A, Pereira-Silva M, Saremnejad F, et al. Naringenin nano-delivery systems and their therapeutic applications. Pharmaceutics. 2021;13, https://doi.org/ 10.3390/pharmaceutics13020291.

38. Zhai K, Brockmüller A, Kubatka P, Shakibaei M, Büsselberg D. Curcumin's beneficial effects on neuroblastoma: mechanisms, challenges, and potential solutions. Biomolecules. 2020;10, https://doi.org/10.3390/biom10111469.

39. Abotaleb M, Liskova A, Kubatka P, Büsselberg D. Therapeutic potential of plant phenolic acids in the treatment of cancer. Biomolecules. 2020;10, doi:https://doi.org/10.3390/biom10020221.

40. Varghese E, Samuel SM, Abotaleb M, Cheema S, Mamtani R, Büsselberg D. The "Yin and Yang" of natural compounds in anticancer therapy of triple-negative breast cancers. Cancers (Basel). 2018;10, https://doi.org/10.3390/cancers10100346.

41. Liskova A, Kubatka P, Samec M, Zubor P, Mlyncek M, Bielik T, et al. Dietary Phytochemicals Targeting Cancer Stem Cells. Molecules 2019;24, https://doi.org/10.3390/molecules24050899.

42. Riganti C, Contino M. New Strategies to overcome resistance to chemotherapy and immune system in cancer. Int J Mol Sci 2019; 20, https://doi.org/10.3390/ijms20194783.

43. Pintova S, Dharmupari S, Moshier E, Zubizarreta N, Ang C, Holcombe RF. Genistein combined with FOLFOX or FOLFOX-Bevacizumab for the treatment of metastatic colorectal cancer: phase I/II pilot study. Cancer Chemother Pharmacol. 2019;84:591-8. https://doi.org/10.1007/s00280-019-03886-3.

44. Lazzeroni M, Guerrieri-Gonzaga A, Gandini S, Johansson H, Serrano D, Cazzaniga M, et al. A presurgical study of lecithin formulation of green tea extract in women with early breast cancer. Cancer Prev Res (Phila). 2017;10:363-70. https://doi.org/10. 1158/1940-6207.CAPR-16-0298.

45. Farsad-Naeimi A, Alizadeh M, Esfahani A, Darvish AE. Effect of fisetin supplementation on inflammatory factors and matrix metalloproteinase enzymes in colorectal cancer patients. Food Funct. 2018;9:2025-31. https://doi.org/10.1039/c7fo01898c.

46. Liskova A, Koklesova L, Samec M, Smejkal K, Samuel SM, Varghese E, et al. Flavonoids in Cancer Metastasis. Cancers (Basel). 2020;12, https://doi.org/10.3390/cancers12061498.

47. Liskova A, Koklesova L, Samec M, Varghese E, Abotaleb M, Samuel SM, et al. Implications of flavonoids as potential modulators of cancer neovascularity. J Cancer Res Clin Oncol. 2020. https://doi.org/10.1007/s00432-020-03383-8.

48. Tang Q, Ma J, Sun J, Yang L, Yang F, Zhang W, et al. Genistein and AG1024 synergistically increase the radiosensitivity of prostate cancer cells. Oncol Rep. 2018;40:579-88. https://doi.org/10. 3892/or.2018.6468.

49. Koh SY, Moon JY, Unno T, Cho SK. Baicalein Suppresses Stem Cell-Like Characteristics in Radio- and Chemoresistant MDAMB-231 Human Breast Cancer Cells through Up-Regulation of IFIT2. Nutrients 2019;11, https://doi.org/10.3390/nu11030624.

50. Li S, Zhao Q, Wang B, Yuan S, Wang X, Li K. Quercetin reversed MDR in breast cancer cells through down-regulating P-gp expression and eliminating cancer stem cells mediated by YB-1 nuclear translocation. Phytother Res. 2018;32:1530-6. https://doi.org/10.1002/ptr.6081.

51. Singh MP, Cho HJ, Kim J-T, Baek KE, Lee HG, Kang SC. Morin hydrate reverses cisplatin resistance by impairing PARP1/ HMGB1-dependent autophagy in hepatocellular carcinoma. Cancers (Basel) 2019;11, https://doi.org/10.3390/cancers11070986. 
52. Riahi-Chebbi I, Souid S, Othman H, Haoues M, Karoui H, Morel A, et al. The Phenolic compound Kaempferol overcomes 5-fluorouracil resistance in human resistant LS174 colon cancer cells. Sci Rep. 2019;9:195. https://doi.org/10.1038/ s41598-018-36808-z.

53. Moradzadeh M, Tabarraei A, Sadeghnia HR, Ghorbani A, Mohamadkhani A, Erfanian S, et al. Kaempferol increases apoptosis in human acute promyelocytic leukemia cells and inhibits multidrug resistance genes. J Cell Biochem. 2018;119:2288-97. https://doi.org/10.1002/jcb.26391.

54. Bae S, D'Cunha R, Shao J, An G. Effect of 5,7-dimethoxyflavone on Bcrp1-mediated transport of sorafenib in vitro and in vivo in mice. Eur J Pharm Sci. 2018;117:27-34. https://doi.org/10. 1016/j.ejps.2018.02.004.

55. Leu Y-L, Wang T-H, Wu C-C, Huang K-Y, Jiang Y-W, Hsu Y-C, et al. Hydroxygenkwanin suppresses non-small cell lung cancer progression by enhancing EGFR degradation. Molecules 2020;25, https://doi.org/10.3390/molecules25040941.

56. Chen Z, Tian D, Liao X, Zhang Y, Xiao J, Chen W, et al. Apigenin combined with gefitinib blocks autophagy flux and induces apoptotic cell death through inhibition of HIF-1 $\alpha$, c-Myc, p-EGFR, and glucose metabolism in EGFR L858R+T790MMutated H1975 Cells. Front Pharmacol. 2019;10:260. https:// doi.org/10.3389/fphar.2019.00260.

57. Sellam LS, Zappasodi R, Chettibi F, Djennaoui D, Yahi-Ait Mesbah N, Amir-Tidadini Z-C, et al. Silibinin down-regulates PD-L1 expression in nasopharyngeal carcinoma by interfering with tumor cell glycolytic metabolism. Arch Biochem Biophys. 2020;690:108479. https://doi.org/10.1016/j.abb.2020.108479.

58. Bailly C. Molecular and cellular basis of the anticancer activity of the prenylated flavonoid icaritin in hepatocellular carcinoma. Chem Biol Interact. 2020;325:109124. https://doi.org/10.1016/j. cbi.2020.109124.

59. Tang H, Liu Y, Wang C, Zheng H, Chen Y, Liu W, et al. Inhibition of COX-2 and EGFR by Melafolone Improves Anti-PD-1 Therapy through Vascular Normalization and PD-L1 Downregulation in Lung Cancer. J Pharmacol Exp Ther. 2019;368:401-13. https://doi.org/10.1124/jpet.118.254359.

60. Nikolaou M, Pavlopoulou A, Georgakilas AG, Kyrodimos E. The challenge of drug resistance in cancer treatment: a current overview. Clin Exp Metastasis. 2018;35:309-18. https://doi.org/ 10.1007/s10585-018-9903-0.

61. Golubnitschaja O, Polivka J, Yeghiazaryan K, Berliner L. Liquid biopsy and multiparametric analysis in management of liver malignancies: new concepts of the patient stratification and prognostic approach. EPMA J. 2018;9:271-85. https://doi.org/ 10.1007/s13167-018-0146-6.

62. Gerner C, Costigliola V, Golubnitschaja O. Multiomic patterns in body fluids: technological challenge with a great potential to implement the advanced paradigm of 3P medicine. Mass Spectrom Rev. 2020;39:442-51. https://doi.org/10.1002/mas.21612.

63. Fröhlich H, Patjoshi S, Yeghiazaryan K, Kehrer C, Kuhn W, Golubnitschaja O. Premenopausal breast cancer: potential clinical utility of a multi-omics based machine learning approach for patient stratification. EPMA J. 2018;9:175-86. https://doi.org/ 10.1007/s13167-018-0131-0.

64. Golubnitschaja O. Feeling cold and other underestimated symptoms in breast cancer: anecdotes or individual profiles for advanced patient stratification? EPMA J. 2017;8:17-22. https:// doi.org/10.1007/s13167-017-0086-6.

65. Goldstein E, Yeghiazaryan K, Ahmad A, Giordano FA, Fröhlich H, Golubnitschaja O. Optimal multiparametric set-up modelled for best survival outcomes in palliative treatment of liver malignancies: unsupervised machine learning and $3 \mathrm{PM}$ recommendations. EPMA J. 2020;1-11, https://doi.org/10.1007/ s13167-020-00221-2.
66. Golubnitschaja O, editor. Flammer syndrome: from phenotype to associated pathologies, prediction, prevention and personalisation advances in predictive, preventive and personalised medicine; Springer International Publishing, 2019; ISBN 978-3-03013549-2, https://doi.org/10.1007/978-3-030-13550-8.

67. Ye Q, Liu K, Shen Q, Li Q, Hao J, Han F, et al. Reversal of Multidrug resistance in cancer by multi-functional flavonoids. Front Oncol 2019;9, https://doi.org/10.3389/fonc.2019.00487.

68. Wong ILK, Zhu X, Chan K-F, Law MC, Lo AMY, Hu X, et al. Discovery of Novel Flavonoid Dimers To Reverse Multidrug Resistance Protein 1 (MRP1, ABCC1) Mediated Drug Resistance in Cancers Using a High Throughput Platform with "Click Chemistry.” J Med Chem. 2018;61:9931-51. https://doi.org/10. 1021/acs.jmedchem.8b00834.

69. Namdeo AG, Boddu SHS, Amawi H, Ashby CR, Tukaramrao DB, Trivedi $\mathrm{P}$, et al. Flavonoids as Multi-Target Compounds: A Special Emphasis on their Potential as Chemo-adjuvants in Cancer Therapy. Curr Pharm Des. 2020;26:1712-28. https://doi. org/10.2174/1381612826666200128095248.

70. Hussain Y, Luqman S, Meena A. Research progress in flavonoids as potential anticancer drug including synergy with other approaches. Curr Top Med Chem. 2020;20:1791-809. https:// doi.org/10.2174/1568026620666200502005411.

71. Abotaleb M, Samuel SM, Varghese E, Varghese S, Kubatka P, Liskova A, et al. Flavonoids in Cancer and Apoptosis. Cancers (Basel) 2018;11, https://doi.org/10.3390/cancers11010028.

72. Samec M, Liskova A, Koklesova L, Mersakova S, Strnadel J, Kajo K, et al. Flavonoids Targeting HIF-1: Implications on Cancer Metabolism. Cancers. 2021;13:130. https://doi.org/10.3390/ cancers 13010130.

73. Lv W-L, Liu Q, An J-H, Song X-Y. Scutellarin inhibits hypoxiainduced epithelial-mesenchymal transition in bladder cancer cells. J Cell Physiol. 2019;234:23169-75. https://doi.org/10. $1002 / j c p .28883$.

74. Hsiao Y-H, Hsieh M-J, Yang S-F, Chen S-P, Tsai W-C, Chen $\mathrm{P}-\mathrm{N}$. Phloretin suppresses metastasis by targeting protease and inhibits cancer stemness and angiogenesis in human cervical cancer cells. Phytomedicine. 2019;62:152964. https://doi.org/ 10.1016/j.phymed.2019.152964.

75. Jiang C-H, Sun T-L, Xiang D-X, Wei S-S, Li W-Q. Anticancer activity and mechanism of xanthohumol: a prenylated flavonoid from hops (Humulus lupulus L.). Front Pharmacol 2018;9, https://doi.org/10.3389/fphar.2018.00530.

76. Kozłowska A, Szostak-Wegierek D. Flavonoids-food sources and health benefits. Rocz Panstw Zakl Hig. 2014;65:79-85.

77. Kumar S, Pandey AK. Chemistry and biological activities of flavonoids: an overview. ScientificWorldJournal 2013;2013, https:// doi.org/10.1155/2013/162750.

78. Panche AN, Diwan AD, Chandra SR. Flavonoids: an overview. J Nutr Sci 2016;5, https://doi.org/10.1017/jns.2016.41.

79. Al-Ishaq RK, Abotaleb M, Kubatka P, Kajo K, Büsselberg D. Flavonoids and their anti-diabetic effects: cellular mechanisms and effects to improve blood sugar levels. Biomolecules. 2019;9, https://doi.org/10.3390/biom9090430.

80. Pichler C, Ferk F, Al-Serori H, Huber W, Jäger W, Waldherr M, et al. Xanthohumol Prevents DNA Damage by Dietary Carcinogens: Results of a Human Intervention Trial. Cancer Prev Res (Phila). 2017;10:153-60. https://doi.org/10.1158/1940-6207. CAPR-15-0378.

81. Lievens Y, Borras JM, Grau C. Provision and use of radiotherapy in Europe. Mol Oncol. 2020;14:1461-9. https://doi.org/10.1002/ 1878-0261.12690.

82. Taguchi K, Yamamoto M. The KEAP1-NRF2 System in Cancer. Front Oncol. 2017;7:85. https://doi.org/10.3389/fonc.2017. 00085 . 
83. Liu X, Sun C, Liu B, Jin X, Li P, Zheng X, et al. Genistein mediates the selective radiosensitizing effect in NSCLC A549 cells via inhibiting methylation of the keap1 gene promoter region. Oncotarget. 2016;7:27267-79. https://doi.org/10.18632/oncot arget.8403.

84. Takahashi A, Kubo M, Ma H, Nakagawa A, Yoshida Y, Isono M, et al. Nonhomologous End-Joining Repair Plays a More Important Role than Homologous Recombination Repair in Defining Radiosensitivity after Exposure to High-LET Radiation. Radiat Res. 2014;182:338-44. https://doi.org/10.1667/RR13782.1.

85. Tiwari P, Mishra KP. Flavonoids sensitize tumor cells to radiation: molecular mechanisms and relevance to cancer radiotherapy. Int J Radiat Biol. 2020;96:360-9. https://doi.org/10.1080/ 09553002.2020.1694193.

86. Zhang Z, Jin F, Lian X, Li M, Wang G, Lan B, et al. Genistein promotes ionizing radiation-induced cell death by reducing cytoplasmic Bcl-xL levels in non-small cell lung cancer. Sci Rep. 2018;8:328. https://doi.org/10.1038/s41598-017-18755-3.

87. Medhat AM, Azab KS, Said MM, El Fatih NM, El Bakary NM. Antitumor and radiosensitizing synergistic effects of apigenin and cryptotanshinone against solid Ehrlich carcinoma in female mice. Tumour Biol. 2017;39:1010428317728480. https://doi.org/10.1177/1010428317728480.

88. Abdraboh ME, Essa ZS, Abdelrazzak AB, El-Far YM, Elsherbini Y, El-Zayat MM, et al. Radio-sensitizing effect of a cocktail of phytochemicals on HepG2 cell proliferation, motility and survival. Biomed Pharmacother. 2020;131:110620. https://doi.org/10.1016/j.biopha.2020.110620.

89. Li Y, Wang Z, Jin J, Zhu S-X, He G-Q, Li S-H, et al. Quercetin pretreatment enhances the radiosensitivity of colon cancer cells by targeting Notch-1 pathway. Biochem Biophys Res Commun. 2020;523:947-53. https://doi.org/10.1016/j.bbrc.2020.01.048.

90. Ma L, Zong X. Metabolic Symbiosis in Chemoresistance: Refocusing the Role of Aerobic Glycolysis. Front Oncol 2020;10, https://doi.org/10.3389/fonc.2020.00005.

91. Wilkens S. Structure and mechanism of ABC transporters. F1000Prime Rep 2015;7, https://doi.org/10.12703/P7-14.

92. Begicevic R-R, Falasca M. ABC Transporters in Cancer Stem Cells: Beyond Chemoresistance. Int J Mol Sci 2017;18, https:// doi.org/10.3390/ijms18112362.

93. Housman G, Byler S, Heerboth S, Lapinska K, Longacre M, Snyder N, et al. Drug Resistance in Cancer: An Overview. Cancers (Basel). 2014;6:1769-92. https://doi.org/10.3390/cance rs6031769.

94. Januchowski R, Wojtowicz K, Zabel M. The role of aldehyde dehydrogenase (ALDH) in cancer drug resistance. Biomed Pharmacother. 2013;67:669-80. https://doi.org/10.1016/j. biopha.2013.04.005.

95. Sakthivel KM, Hariharan S. Regulatory players of DNA damage repair mechanisms: Role in Cancer Chemoresistance. Biomed Pharmacother. 2017;93:1238-45. https://doi.org/10. 1016/j.biopha.2017.07.035

96. Salehan MR, Morse HR. DNA damage repair and tolerance: a role in chemotherapeutic drug resistance. Br J Biomed Sci. 2013;70:31-40. https://doi.org/10.1080/09674845.2013.11669 927.

97. Liu R, Chen Y, Liu G, Li C, Song Y, Cao Z, et al. PI3K/AKT pathway as a key link modulates the multidrug resistance of cancers. Cell Death Dis 2020;11, https://doi.org/10.1038/ s41419-020-02998-6.

98. Lu X, Yang F, Chen D, Zhao Q, Chen D, Ping H, et al. Quercetin reverses docetaxel resistance in prostate cancer via androgen receptor and PI3K/Akt signaling pathways. Int J Biol Sci. 2020;16:1121-34. https://doi.org/10.7150/ijbs.41686.

99. Choi HJ, Heo JH, Park JY, Jeong JY, Cho HJ, Park KS, et al. A novel PI3K/mTOR dual inhibitor, CMG002, overcomes the chemoresistance in ovarian cancer. Gynecol Oncol. 2019;153:135-48. https://doi.org/10.1016/j.ygyno.2019.01. 012.

100. Cho Y, Kim YK. Cancer Stem Cells as a Potential Target to Overcome Multidrug Resistance. Front Oncol 2020;10, https:// doi.org/10.3389/fonc.2020.00764.

101. Bahar E, Kim J-Y, Yoon H. Chemotherapy Resistance Explained through Endoplasmic Reticulum Stress-Dependent Signaling. Cancers (Basel) 2019, 11, doi:https://doi.org/10.3390/cancers110 30338.

102. Kim E-K, Jang M, Song M-J, Kim D, Kim Y, Jang HH. Redoxmediated mechanism of chemoresistance in cancer cells. Antioxidants (Basel). 2019;8, https://doi.org/10.3390/antiox8100471.

103. Lan C-Y, Chen S-Y, Kuo C-W, Lu C-C, Yen G-C. Quercetin facilitates cell death and chemosensitivity through RAGE/PI3K/ AKT/mTOR axis in human pancreatic cancer cells. J Food Drug Anal. 2019;27:887-96. https://doi.org/10.1016/j.jfda.2019.07. 001.

104. Hassanzadeh P. Colorectal cancer and NF- $\kappa B$ signaling pathway. Gastroenterol Hepatol Bed Bench. 2011;4:127-32, PMC4017424.

105. Liu S, Li R, Qian J, Sun J, Li G, Shen J, et al. Combination therapy of doxorubicin and quercetin on multidrug-resistant breast cancer and their sequential delivery by reduction-sensitive hyaluronic acid-based conjugate/d- $\alpha$-Tocopheryl Poly(ethylene glycol) 1000 succinate mixed micelles. Mol Pharm. 2020;17:1415-27. https://doi.org/10.1021/acs.molpharmaceut.0c00138.

106. Bieg D, Sypniewski D, Nowak E, Bednarek I. Morin decreases galectin-3 expression and sensitizes ovarian cancer cells to cisplatin. Arch Gynecol Obstet. 2018;298:1181-94. https://doi.org/ 10.1007/s00404-018-4912-4.

107. Zhang X, Huang J, Yu C, Xiang L, Li L, Shi D, et al. Quercetin Enhanced Paclitaxel Therapeutic Effects Towards PC-3 Prostate Cancer Through ER Stress Induction and ROS Production. Onco Targets Ther. 2020;13:513-23. https://doi.org/10.2147/OTT. S228453.

108. Silbermann K, Shah CP, Sahu NU, Juvale K, Stefan SM, Kharkar PS, et al. Novel chalcone and flavone derivatives as selective and dual inhibitors of the transport proteins $\mathrm{ABCB} 1$ and $\mathrm{ABCG} 2$. Eur J Med Chem. 2019;164:193-213. https://doi.org/10.1016/j. ejmech.2018.12.019.

109. Kong W, Ling X, Chen Y, Wu X, Zhao Z, Wang W, et al. Hesperetin reverses P-glycoprotein-mediated cisplatin resistance in DDP-resistant human lung cancer cells via modulation of the nuclear factor- $\mathrm{\kappa B}$ signaling pathway. Int J Mol Med. 2020;45:1213-24. https://doi.org/10.3892/ijmm.2020.4485.

110. Zhao L, Zhang W, Zhang F. Poncirin downregulates ATP-binding cassette transporters to enhance cisplatin sensitivity in cisplatinresistant osteosarcoma cells. Phytother Res. 2021;35:278-88. https://doi.org/10.1002/ptr.6798.

111. Scagliarini A, Mathey A, Aires V, Delmas D. Xanthohumol, a Prenylated Flavonoid from Hops, Induces DNA Damages in Colorectal Cancer Cells and Sensitizes SW480 Cells to the SN38 Chemotherapeutic Agent. Cells. 2020;9, https://doi.org/10.3390/ cells9040932.

112. Hua R, Pei Y, Gu H, Sun Y, He Y. Antitumor effects of flavokawain-B flavonoid in gemcitabine-resistant lung cancer cells are mediated via mitochondrial-mediated apoptosis, ROS production, cell migration and cell invasion inhibition and blocking of PI3K/AKT Signaling pathway. J BUON. 2020;25:262-7.

113. Fan X, Bai J, Zhao S, Hu M, Sun Y, Wang B, et al. Evaluation of inhibitory effects of flavonoids on breast cancer resistance protein (BCRP): From library screening to biological evaluation to structure-activity relationship. Toxicol In Vitro. 2019;61:104642. https://doi.org/10.1016/j.tiv.2019.104642. 
114. Wang Z, Sun X, Feng Y, Wang Y, Zhang L, Wang Y, et al. Dihydromyricetin reverses MRP2-induced multidrug resistance by preventing NF- $\mathrm{\kappa B}-\mathrm{Nrf} 2$ signaling in colorectal cancer cell. Phytomedicine. 2021;82:153414. https://doi.org/10.1016/j.phymed. 2020.153414.

115. Darzi S, Mirzaei SA, Elahian F, Shirian S, Peymani A, Rahmani $\mathrm{B}$, et al. Enhancing the Therapeutic Efficacy of Daunorubicin and Mitoxantrone with Bavachinin, Candidone, and Tephrosin. Evid Based Complement Alternat Med. 2019;2019:3291737. https:// doi.org/10.1155/2019/3291737.

116. Khonkarn R, Daowtak K, Okonogi S. Chemotherapeutic Efficacy Enhancement in P-gp-Overexpressing Cancer Cells by Flavonoid-Loaded Polymeric Micelles. AAPS PharmSciTech. 2020;21:121. https://doi.org/10.1208/s12249-020-01657-5.

117. Jabri T, Imran M, Aziz A, Rao K, Kawish M, Irfan M, et al. Design and synthesis of mixed micellar system for enhanced anticancer efficacy of Paclitaxel through its co-delivery with Naringin. Drug Dev Ind Pharm. 2019;45:703-14. https://doi.org/10. 1080/03639045.2018.1550091.

118. Wang B, Guo C, Liu Y, Han G, Li Y, Zhang Y, et al. Novel nano-pomegranates based on astragalus polysaccharides for targeting ER $\alpha$-positive breast cancer and multidrug resistance. Drug Deliv. 2020;27:607-21. https://doi.org/10.1080/10717 544.2020.1754529.

119. Wen M, Xia J, Sun Y, Wang X, Fu X, Zhang Y, et al. Combination of EGFR-TKIs with chemotherapy versus chemotherapy or EGFR-TKIs alone in advanced NSCLC patients with EGFR mutation. Biologics. 2018;12:183-90. https://doi.org/10.2147/ BTT.S169305.

120. Karachaliou N, Gonzalez-Cao M, Sosa A, Berenguer J, Bracht JWP, Ito M, et al. The combination of checkpoint immunotherapy and targeted therapy in cancer. Ann Transl Med 2017;5, https://doi.org/10.21037/atm.2017.06.47.

121. Wang H, Pan R, Zhang X, Si X, Wang M, Zhang L. Abivertinib in patients with T790M-positive advanced NSCLC and its subsequent treatment with osimertinib. Thorac Cancer. 2020;11:594-602. https://doi.org/10.1111/1759-7714.13302.

122. Huang S, Yu M, Shi N, Zhou Y, Li F, Li X, et al. Apigenin and Abivertinib, a novel BTK inhibitor synergize to inhibit diffuse large B-cell lymphoma in vivo and vitro. J Cancer. 2020;11:2123-32. https://doi.org/10.7150/jca.34981.

123. Harrison PT, Huang PH. Exploiting vulnerabilities in cancer signalling networks to combat targeted therapy resistance. Essays Biochem. 2018;62:583-93. https://doi.org/10.1042/ EBC20180016.

124. Sambi M, Szewczuk MR. Introduction to the Acquisition of Resistance to Targeted Therapy. In: Szewczuk MR, Qorri B, Sambi M, editors. Current Applications for Overcoming Resistance to Targeted Therapies, Springer International Publishing: Cham, 2019; pp. 1-33 ISBN 978-3-030-21477-7, https://doi.org/10.1007/978-3-030-21477-7_1.

125. Sabnis AJ, Bivona TG. Principles of Resistance to Targeted Cancer Therapy: Lessons from Basic and Translational Cancer Biology. Trends in Molecular Medicine. Elsevier; 2019;25:185-97, https://doi.org/10.1016/j.molmed.2018.12. 009 .

126. Groenendijk FH, Bernards R. Drug resistance to targeted therapies: Déjà vu all over again. Mol Oncol. 2014;8:1067-83. https://doi.org/10.1016/j.molonc.2014.05.004.

127. Chen D-H, Zhang X-S. Targeted therapy: resistance and resensitization. Chin J Cancer 2015;34, https://doi.org/10.1186/ s40880-015-0047-1.

128. Li X, Xu J, Tang X, Liu Y, Yu X, Wang Z, et al. Anthocyanins inhibit trastuzumab-resistant breast cancer in vitro and in vivo. Mol Med Rep. 2016;13:4007-13. https://doi.org/10.3892/mmr. 2016.4990 .
129. Zhang L, Qi Y, Xing K, Qian S, Zhang P, Wu X. A novel strategy of EGFR-TKI combined chemotherapy in the treatment of human lung cancer with EGFR-sensitive mutation. Oncology Reports. Spandidos Publications; 2018;40:1046-54, https://doi.org/10. 3892/or.2018.6499.

130. Sim EH, Yang IA, Wood-Baker R, Bowman RV, Fong KM. Gefitinib for advanced non-small cell lung cancer. Cochrane Database Syst Rev. 2018;1:CD006847. https://doi.org/10.1002/14651858. CD006847.pub2.

131. Wu Z, Xu B, Yu Z, He Q, Hu Z, Zhou S, et al. Trifolium Flavonoids Overcome Gefitinib Resistance of Non-Small-Cell Lung Cancer Cell by Suppressing ERK and STAT3 Signaling Pathways. Biomed Res Int. 2020;2020:2491304. https://doi.org/10. 1155/2020/2491304

132. Liu J, Zhong T, Yi P, Fan C, Zhang Z, Liang G, et al. A new epigallocatechin gallate derivative isolated from Anhua dark tea sensitizes the chemosensitivity of gefitinib via the suppression of $\mathrm{PI} 3 \mathrm{~K} / \mathrm{mTOR}$ and epithelial-mesenchymal transition. Fitoterapia. 2020;143:104590. https://doi.org/10.1016/j.fitote.2020.104590.

133. Tang W, Chen Z, Zhang W, Cheng Y, Zhang B, Wu F, et al. The mechanisms of sorafenib resistance in hepatocellular carcinoma: theoretical basis and therapeutic aspects. Signal Transduction and Targeted Therapy. Nature Publishing Group; 2020;5:1-15, https://doi.org/10.1038/s41392-020-0187-x.

134. Li B, Feng F, Jia H, Jiang Q, Cao S, Wei L, et al. Rhamnetin decelerates the elimination and enhances the antitumor effect of the molecular-targeting agent sorafenib in hepatocellular carcinoma cells via the miR-148a/PXR axis. Food Funct. 2021. https://doi.org/10.1039/d0fo02270e.

135. Şirin N, Elmas L, Seçme M, Dodurga Y. Investigation of possible effects of apigenin, sorafenib and combined applications on apoptosis and cell cycle in hepatocellular cancer cells. Gene. 2020;737:144428. https://doi.org/10.1016/j.gene.2020.144428.

136. Saraswati S, Alhaider A, Abdelgadir AM, Tanwer P, Korashy HM. Phloretin attenuates STAT-3 activity and overcomes sorafenib resistance targeting SHP-1-mediated inhibition of STAT3 and Akt/VEGFR2 pathway in hepatocellular carcinoma. Cell Commun Signal. 2019;17:127. https://doi.org/10.1186/ s12964-019-0430-7.

137. Nair B, Anto RJ, M S, Nath LR. Kaempferol-Mediated Sensitization Enhances Chemotherapeutic Efficacy of Sorafenib Against Hepatocellular Carcinoma: An In Silico and In Vitro Approach. Adv Pharm Bull. 2020;10:472-6, https://doi.org/10.34172/apb. 2020.058

138. Yeh B-W, Yu L-E, Li C-C, Yang J-C, Li W-M, Wu Y-C, et al. The protoapigenone analog WYC0209 targets CD133+ cells: A potential adjuvant agent against cancer stem cells in urothelial cancer therapy. Toxicol Appl Pharmacol. 2020;402:115129. https://doi.org/10.1016/j.taap.2020.115129.

139. Li L, Zheng Y, Zhang W, Hou L, Gao Y. Scutellarin circumvents chemoresistance, promotes apoptosis, and represses tumor growth by HDAC/miR-34a-mediated down-modulation of Akt/ $\mathrm{mTOR}$ and NF- $\mathrm{KB}$-orchestrated signaling pathways in multiple myeloma. Int J Clin Exp Pathol. 2020;13:212-9. PMC7061792.

140. Kim B, Seo JH, Lee KY, Park B. Icariin sensitizes human colon cancer cells to TRAIL-induced apoptosis via ERK-mediated upregulation of death receptors. Int J Oncol. 2020;56:821-34. https://doi.org/10.3892/ijo.2020.4970.

141. Liu Y-S, Yu-Chun, Chang, Kuo W-W, Chen M-C, Hsu H-H, et al. Inhibition of protein phosphatase 1 stimulates noncanonical ER stress eIF $2 \alpha$ activation to enhance fisetin-induced chemosensitivity in HDAC inhibitor-resistant hepatocellular carcinoma cells. Cancers (Basel). 2019;11, https://doi.org/10.3390/cancers110 70918.

142. Suraweera A, O'Byrne KJ, Richard DJ. Combination Therapy With Histone Deacetylase Inhibitors (HDACi) for the Treatment 
of Cancer: Achieving the Full Therapeutic Potential of HDACi. Front Oncol 2018;8, https://doi.org/10.3389/fonc.2018.00092.

143. Thapa B, Kc R, Uludağ H. TRAIL therapy and prospective developments for cancer treatment. J Control Release. 2020;326:33549. https://doi.org/10.1016/j.jconrel.2020.07.013.

144. Wang F, Lin J, Xu R. The molecular mechanisms of TRAIL resistance in cancer cells: help in designing new drugs. Curr Pharm Des. 2014;20:6714-22. https://doi.org/10.2174/13816 12820666140929100735.

145. Guo S, Zhang Y, Wu Z, Zhang L, He D, Li X, et al. Synergistic combination therapy of lung cancer: Cetuximab functionalized nanostructured lipid carriers for the co-delivery of paclitaxel and 5-Demethylnobiletin. Biomed Pharmacother. 2019;118:109225. https://doi.org/10.1016/j.biopha.2019.109225.

146. Draghi A, Chamberlain CA, Furness A, Donia M. Acquired resistance to cancer immunotherapy. Semin Immunopathol. 2019;41:31-40. https://doi.org/10.1007/s00281-018-0692-y.

147. Gou Q, Dong C, Xu H, Khan B, Jin J, Liu Q, et al. PD-L1 degradation pathway and immunotherapy for cancer. Cell Death \& Disease. Nature Publishing Group; 2020;11:1-7, https://doi.org/ 10.1038/s41419-020-03140-2.

148. Nowicki TS, Hu-Lieskovan S, Ribas A. Mechanisms of Resistance to PD-1 and PD-L1 blockade. Cancer J. 2018;24:47-53. https://doi.org/10.1097/PPO.0000000000000303.

149. Furukawa K, Nagano T, Tachihara M, Yamamoto M, Nishimura Y. Interaction between Immunotherapy and Antiangiogenic Therapy for Cancer. Molecules 2020;25, https://doi.org/10.3390/ molecules25173900.

150. Yin S-Y, Yang N-S, Lin T-J. Phytochemicals Approach for Developing Cancer Immunotherapeutics. Front Pharmacol 2017;8, https://doi.org/10.3389/fphar.2017.00386.
151. Kopustinskiene DM, Jakstas V, Savickas A, Bernatoniene J. Flavonoids as Anticancer Agents. Nutrients 2020;12, https://doi.org/ 10.3390/nu12020457.

152. Kumar AR, Devan AR, Nair B, Nath LR. Anti-VEGF Mediated Immunomodulatory Role of Phytochemicals: Scientific Exposition for Plausible HCC Treatment. Curr Drug Targets. 2021. https://doi.org/10.2174/1389450122666210203194036.

153. Awan FT, Jones JA, Maddocks K, Poi M, Grever MR, Johnson A, et al. A phase 1 clinical trial of flavopiridol consolidation in chronic lymphocytic leukemia patients following chemoimmunotherapy. Ann Hematol. 2016;95:1137-43. https://doi.org/10. 1007/s00277-016-2683-1.

154. Fan Y, Li S, Ding X, Yue J, Jiang J, Zhao H, et al. First-in-class immune-modulating small molecule Icaritin in advanced hepatocellular carcinoma: preliminary results of safety, durable survival and immune biomarkers. BMC Cancer. 2019;19:279. https://doi org/10.1186/s12885-019-5471-1.

155. Xu L, Zhang Y, Tian K, Chen X, Zhang R, Mu X, et al. Apigenin suppresses PD-L1 expression in melanoma and host dendritic cells to elicit synergistic therapeutic effects. J Exp Clin Cancer Res 2018;37, https://doi.org/10.1186/s13046-018-0929-6.

156. Cimini A, d'Angelo M, Benedetti E, D'Angelo B, Laurenti G, Antonosante A, et al. Flavopiridol: An Old Drug With New Perspectives? Implication for Development of New Drugs. J Cell Physiol. 2017;232:312-22. https://doi.org/10.1002/jcp.25421.

Publisher's note Springer Nature remains neutral with regard to jurisdictional claims in published maps and institutional affiliations.

\section{Authors and Affiliations}

\section{Alena Liskova ${ }^{1} \cdot$ Marek Samec $^{1} \cdot$ Lenka Koklesova $^{1} \cdot$ Aranka Brockmueller $^{2} \cdot$ Kevin $_{\text {Zhai }}{ }^{3} \cdot$ Basma Abdellatif $^{3}$. Manaal Siddiqui ${ }^{3} \cdot$ Kamil Biringer $^{1} \cdot$ Erik Kudela $^{1} \cdot$ Martin Pec $^{4} \cdot$ Laura Kate Gadanec $^{5} \cdot$ Miroslava Šudomová $^{6}$. Sherif T. S. Hassan ${ }^{7}$. Anthony Zulli ${ }^{5} \cdot$ Mehdi Shakibaei $^{2}$. Frank A. Giordano ${ }^{8}$ - Dietrich Büsselberg ${ }^{3}$. Olga Golubnitschaja ${ }^{9} \cdot$ Peter Kubatka ${ }^{4}$}

1 Department of Obstetrics and Gynecology, Jessenius Faculty of Medicine, Comenius University in Bratislava, 03601 Martin, Slovakia

2 Musculoskeletal Research Group and Tumor Biology, Chair of Vegetative Anatomy, Institute of Anatomy, Faculty of Medicine, Ludwig-Maximilian-University Munich, 80336 Munich, Germany

3 Weill Cornell Medicine-Qatar, Education City, Qatar Foundation, 24144 Doha, Qatar

4 Department of Medical Biology, Jessenius Faculty of Medicine, Comenius University in Bratislava, 03601 Martin, Slovakia

5 Institute for Health and Sport, Victoria University, Melbourne 3030, Australia
6 Museum of Literature in Moravia, Klášter 1, 66461 Rajhrad, Czech Republic

7 Department of Applied Ecology, Faculty of Environmental Sciences, Czech University of Life Sciences Prague, Kamýcká 129, 16500 Prague, Czech Republic

8 Department of Radiation Oncology, University Hospital Bonn, Rheinische Friedrich-Wilhelms-Universität Bonn, Bonn, Germany

9 Predictive, Preventive and Personalised (3P) Medicine, Department of Radiation Oncology, University Hospital Bonn, Rheinische Friedrich-Wilhelms-Universität Bonn, 53127 Bonn, Germany 